Pré-Publicações do Departamento de Matemática

Universidade de Coimbra

Preprint Number 03-07

\title{
ON GENERALIZED NUMERICAL RANGES OF OPERATORS ON AN INDEFINITE INNER PRODUCT SPACE
}

\author{
N. BEBIANO, R. LEMOS, J. DA PROVIDÊNCIA AND G. SOARES
}

\begin{abstract}
In this paper, numerical ranges associated to operators on an indefinite inner product space are investigated. Boundary generating curves, corners, shapes and computer generations of these sets are studied. In particular, the MurnaghanKippenhahn theorem for the classical numerical range is generalized.
\end{abstract}

KEYWORDS: numerical range, generalized numerical range, indefinite inner product space.

AMS SubJeCt ClassificAtion (2000): 15A60, 15A63, 15A45.

\section{The $S, C$-Tracial Range and the $S, C$-Determinantal Range}

Let $A \in M_{n}$, the algebra of $n \times n$ complex matrices, and $S \in M_{n}$ be a Hermitian matrix. The $S$-numerical range of $A$ is denoted and defined by

$$
V_{S}(A)=\left\{\frac{x^{*} A x}{x^{*} S x}: x \in \mathbb{C}^{n}, x^{*} S x \neq 0\right\} .
$$

For convenience, consider the sets

$$
V_{S}^{ \pm}(A)=\left\{x^{*} A x: x \in \mathbb{C}^{n}, x^{*} S x= \pm 1\right\},
$$

which have been studied by other researchers $[6,12]$. It is easy to verify that

$$
V_{-S}^{+}(A)=V_{S}^{-}(A) \quad \text { and } \quad V_{S}(A)=V_{S}^{+}(A) \cup-V_{-S}^{+}(A) .
$$

If $S$ is the identity matrix $I_{n}$, then $V_{S}^{-}(A)$ is the empty set and the $S$ numerical range $V_{S}(A)=V_{S}^{+}(A)$ reduces to the classical numerical range, usually denoted by $W(A)$.

The sets $W_{S}(A)=V_{S}(S A)$ and $W_{S}^{ \pm}(A)=V_{S}^{ \pm}(S A)$ have also been investigated. When $S$ is a nonsingular indefinite Hermitian matrix, some authors use $W_{S}(A)$ or $W_{S}^{+}(A)$ as the definition for a numerical range of $A$ associated with the indefinite inner product $\langle x, y\rangle_{S}=y^{*} S x$. In [12, 13, 14], these 
sets were investigated in connection with the cone generated by the joint numerical range of three Hermitian matrices $(H, G, S)$ :

$$
W(H, G, S)=\left\{\left(x^{*} H x, x^{*} G x, x^{*} S x\right): x \in \mathbb{C}^{n}, x^{*} x=1\right\},
$$

where $S A=H+i G$. Our study does not follow such an approach. Instead, we focus directly on the concepts under investigation.

We list some basic properties of the $S$-numerical range that follow easily from the definition:

P1. $V_{S}(A)=V_{S}\left(U^{*} A U\right)$, for any matrix $U \in M_{n}$ and any nonsingular Hermitian $S$ such that $U^{*} S U=S$.

P2. $V_{S}(\alpha S+\beta A)=\alpha+\beta V_{S}(A)$, for any $\alpha, \beta \in \mathbb{C}$.

P3. $V_{S}\left(A^{*}\right)=\overline{V_{S}(A)}$.

P4. $V_{S}(A+B) \subset V_{S}(A)+V_{S}(B)$.

P5. $V_{S}(A)=\{\lambda\}$ if and only if $S \neq 0$ and $A=\lambda S$, for $\lambda \in \mathbb{C}$, that is, $A$ is a $S$-scalar matrix.

P6. $V_{S}(A) \subseteq \mathbb{R}$ if and only if $A$ is Hermitian.

The same properties are valid when $V_{S}$ is replaced by $V_{S}^{ \pm}$, except P2 and P5. For these sets, the following properties hold:

P2'. $V_{S}^{ \pm}(\alpha S+\beta A)= \pm \alpha+\beta V_{S}^{ \pm}(A)$, for any $\alpha, \beta \in \mathbb{C}$.

P5'. $V_{S}^{ \pm}(A)=\{\lambda\}$ if and only if $A= \pm \lambda S$, for $\lambda \in \mathbb{C}$, and $S$ has at least one positive (or negative) eigenvalue.

Denote by $\sigma_{S}(A)$ the set of the eigenvalues of $A$ that have $S$-anisotropic eigenvectors, that is, vectors $x$ for which $x^{*} S x \neq 0$. For $S$ invertible, we obtain:

P7. $\sigma_{S}\left(S^{-1} A\right) \subset V_{S}(A)$.

If $S=I_{n}$, then $\sigma_{S}\left(S^{-1} A\right)=\sigma(A)$, the spectrum of $A$, and the previous property reduces to the spectral inclusion of the classical numerical range.

The classical numerical range $W(A)$ is a compact set. In contrast with the classical case, when $S$ is nonsingular indefinite and $A$ is not a $S$-scalar matrix, the set $V_{S}(A)$ is unbounded and may not be closed $[12,13]$.

By the celebrated Toeplitz-Hausdorff theorem, $W(A)$ is a convex set [8]. The sets $V_{S}^{ \pm}(A)$ are also convex; however $V_{S}(A)$ may not be convex. Nevertheless, $V_{S}(A)$ is $p$-convex [12]; that is, for any pair of distinct points $x, y \in V_{S}(A)$, either $V_{S}(A)$ contains the closed line segment joining $x$ and 
$y$, or $V_{S}(A)$ contains the line defined by $x$ and $y$, except the open line segment joining $x$ and $y$.

Motivated by theory and applications, there are several generalizations of the classical numerical range, such as the $C$-numerical range of $A$ denoted by $W_{C}(A)$. For $A, C \in M_{n}, W_{C}(A)$ is defined by

$$
W_{C}(A)=\left\{\operatorname{Tr}\left(C U^{*} A U\right): U \in M_{n}, U^{*} U=I\right\} .
$$

This concept motivates the definition of the $S, C$-tracial range of $A$ for $A, C \in$ $M_{n}$ :

$$
V_{S, C}(A)=\left\{\operatorname{Tr}\left(C U^{*} A U\right): U \in M_{n}, U^{*} S U=S\right\} .
$$

The following properties of the $S, C$-tracial range are easily deduced:

Q1. $V_{S, C}(A)=V_{S, C}\left(U^{*} A U\right)$, for any matrix $U \in M_{n}$ and any nonsingular Hermitian $S$ such that $U^{*} S U=S$.

Q2. $V_{S, C}(\alpha S+\beta A)=\alpha \operatorname{Tr}(S C)+\beta V_{S, C}(A)$, for any $\alpha, \beta \in \mathbb{C}$.

Q3. $V_{S, C^{*}}\left(A^{*}\right)=\overline{V_{S, C}(A)}$.

Q4. $V_{S, C}(A)=V_{S^{-1}, A}(C)$, for $S$ nonsingular Hermitian.

As a variation of (1), for $A, C \in M_{n}$ we define the $S, C$-determinantal range of $A$ :

$$
D_{S, C}(A)=\left\{\operatorname{det}\left(C+U^{*} A U\right): U \in M_{n}, U^{*} S U=S\right\} .
$$

When $S=I_{n}$, this concept reduces to the $C$-determinantal range $\triangle_{C}(A)$ [2].

The following properties of the $S, C$-determinantal range can be easily verified:

R1. $D_{S, C}(A)=D_{S, C}\left(U^{*} A U\right)$, for any matrix $U \in M_{n}$ and any nonsingular Hermitian $S$ such that $U^{*} S U=S$.

R2. $D_{S, C^{*}}\left(A^{*}\right)=\overline{D_{S, C}(A)}$.

R3. $D_{S, C}(A)=D_{S, A}(C)$, for $S$ nonsingular Hermitian, that is, the roles of $A$ and $C$ are symmetric.

When $C=\operatorname{diag}\left(\gamma_{1}, \ldots, \gamma_{n}\right), W_{C}(A)$ and $\triangle_{C}(A)$ are usually denoted by $W_{c}(A)$ and $\triangle_{c}(A)$, respectively, where $c=\left(\gamma_{1}, \ldots, \gamma_{n}\right) \in \mathbb{C}^{n}$. The notations $V_{S, c}(A)$ and $\triangle_{S, c}(A)$ will be used in a similar way.

A matrix $U \in M_{n}$ is pseudo-unitary of signature $(r, n-r)$, with $0 \leq r \leq n$, if the corresponding linear transformation preserves the quadratic Hermitian 
form

$$
q(x)=\left|x_{1}\right|^{2}+\cdots+\left|x_{r}\right|^{2}-\left|x_{r+1}\right|^{2}-\cdots-\left|x_{n}\right|^{2} .
$$

The group of pseudo-unitary matrices of signature $(r, n-r)$ will be denoted by $U_{r, n-r}$.

Let $J=P\left(I_{r} \oplus-I_{n-r}\right) P^{T}$, where $P$ is a permutation matrix. It can be easily seen that $U=\left[x_{1} \cdots x_{n}\right]$, where $x_{j}$ denotes the $j$ th column of $U$, is a matrix in the pseudo-unitary group $U_{r, n-r}$ if and only if $U^{*} J U=J$, that is,

$$
x_{i}^{*} J x_{k}=0, \quad i \neq k, \quad \text { and } \quad x_{i}^{*} J x_{i}=j_{i}, \quad i, k=1, \ldots, n,
$$

where $j_{i}$ denotes the $(i, i)$ entry of the matrix $J$.

If $c=\left(\gamma_{1}, \ldots, \gamma_{n}\right) \in \mathbb{C}^{n}$, then we obtain directly from the definition of the $J, c$-tracial range

$$
V_{J, c}(A)=\left\{\sum_{i=1}^{n} \gamma_{i} x_{i}^{*} A x_{i}: x_{i} \in \mathbb{C}^{n} \text { satisfying (2) }\right\} .
$$

If $c=e_{i}$ denotes the $i$ th vector of the standard basis of $\mathbb{C}^{n}$, it follows that $V_{J, e_{i}}(A)$ reduces to $V_{J}^{ \pm}(A)$, according to $j_{i}= \pm 1$.

Since the pseudo-unitary group $U_{r, n-r}$ is connected and $V_{J, C}(A)\left(D_{J, C}(A)\right)$ is the range of the continuous mapping from $U_{r, n-r}$ to $\mathbb{C}$ defined by $U \mapsto$ $\operatorname{Tr}\left(C U^{*} A U\right)\left(U \mapsto \operatorname{det}\left(C+U^{*} A U\right)\right), V_{J, C}(A)\left(D_{J, C}(A)\right)$ is a connected set, for any $A, C \in M_{n}$.

In this paper, we assume that the Hermitian matrix $S$ is nonsingular and we observe that, in this case, it is not a restriction to consider the matrix $J$ instead of $S$ in the definition of the $S$-numerical range. In fact, recalling Sylvester's law of inertia [7], we can always choose a nonsingular matrix $R$, such that $R^{*} S R=I_{r} \oplus-I_{n-r}$, the inertia matrix of $S$. Considering $y=R^{-1} x$ and $A_{R}=R^{*} A R$, we have

$$
\frac{x^{*} A x}{x^{*} S x}=\frac{y^{*} A_{R} y}{y^{*} J y}
$$

and this means that

$$
V_{S}(A)=V_{J}\left(A_{R}\right)
$$

for $J$ the inertia matrix of $S$. With respect to the $S, C$-tracial range and the $S, C$-determinantal range, using again Sylvester's law of inertia, we can 
easily check that the following relations hold:

$$
V_{S, C}(A)=V_{J, C_{\left(R^{*}\right)-1}}\left(A_{R}\right) \quad \text { and } \quad D_{S, C}(A)=|\operatorname{det} R|^{-2} D_{J, C_{R}}\left(A_{R}\right),
$$

where $C_{R}=R^{*} C R$.

The paper is organized as follows. In section 2 , the equation in line coordinates of the boundary generating curve of $V_{S}(A)$ is obtained. This is a generalization of a result due to Murnaghan and Kippenhahn $[11,16]$ concerning the classical numerical range. Using a theorem of Tarski, we prove that the boundaries of $V_{S, C}(A)$ and $D_{S, C}(A)$ are a finite union of algebraic arcs. In section 3 , the hyperbolical range theorem is revisited and alternative proofs to the one in [13] are given. In section 4 , the $J, c$-tracial range and the $J, c$-determinantal range of arbitrary $2 \times 2$ matrices are described. In section 5 , the matrices $A$ for which $V_{J, c}(A)$ is a singleton or a subset of a line are characterized. In section 6 , an analogous study is done for $D_{J, c}(A)$, for diagonal matrices $A$. In section 7 , some special boundary points (the corners) of $V_{J, c}(A)$ and $D_{J, c}(A)$ are investigated. Finally, in section 8, a Matlab program is developed to generate $V_{J}(A)$.

\section{The Boundary Generating Curve of $V_{S}(A)$}

Let $z \in V_{S}(A)$ be a boundary point of $V_{S}(A)$. A line containing $z$ and defining two half planes, such that one of them does not contain $V_{S}^{+}(A)$ or $-V_{S}^{-}(A)$ will be called a support line of $V_{S}(A)$.

For any matrix $A, \operatorname{Re} A$ will denote the Hermitian matrix $\left(A+A^{*}\right) / 2$. Throughout the paper, the following result will be used.

Proposition 2.1. Let $A \in M_{n}$ and $\theta \in \mathbb{R}$. If the eigenvalues of $J \operatorname{Re}\left(e^{i \theta} A\right)$ are not all real, then $V_{J}(A)$ has not a support line in the direction $\theta$.

Proof: For simplicity, denote $\operatorname{Re}\left(e^{i \theta} A\right)$ by $A^{\prime}$. It can be easily seen that if $\lambda$ is a complex eigenvalue of $J A^{\prime}$, then $\bar{\lambda}$ also is. Now, we assume that the eigenvalues of $J A^{\prime}$ are all simple. Under this hypothesis, there exists a full eigenbasis of $J A^{\prime}$, say $f_{1}, \ldots, f_{n}$.

Denote by $D \in M_{n}$ the matrix whose $j$ th column is $f_{j}$. It can be shown that if $f, f^{\prime}$ are vectors of this eigenbasis, $A^{\prime} f=\lambda J f$, and $A^{\prime} f^{\prime}=\lambda^{\prime} J f^{\prime}$, with $0 \neq f, f^{\prime} \in \mathbb{C}^{n}$ and $\lambda \neq \bar{\lambda}^{\prime}$, then $f^{*} J f^{\prime}=0$. Let $\lambda \in \mathbb{C}$ and let $0 \neq u, v \in \mathbb{C}^{n}$ be vectors of this eigenbasis such that $A^{\prime} u=\lambda J u, A^{\prime} v=\bar{\lambda} J v$ and $u^{*} J v=\gamma$. Hence, the matrix $D^{*} J D$ is a diagonal block matrix of $2 \times 2$ and $1 \times 1$ blocks. 
The $2 \times 2$ blocks correspond to complex eigenvalues and have zero diagonal entries while the $1 \times 1$ blocks correspond to real eingenvalues. Obviously, $\operatorname{det}\left(D^{*} J D\right) \neq 0$ and this implies that $\gamma \neq 0$.

For the above $u, v$, it is clear that $u^{*} A^{\prime} u=v^{*} A^{\prime} v=0, u^{*} A^{\prime} v=\bar{\lambda} \gamma$ and $v^{*} A^{\prime} u=\lambda \bar{\gamma}$. Consider the subset $T_{u, v}$ of $V_{J}\left(A^{\prime}\right)$ defined by

$$
T_{u, v}=\left\{\frac{\left(u+v e^{i \phi}\right)^{*} A^{\prime}\left(u+v e^{i \phi}\right)}{\left(u+v e^{i \phi}\right)^{*} J\left(u+v e^{i \phi}\right)}: \phi \in \mathbb{R},\left(u+v e^{i \phi}\right)^{*} J\left(u+v e^{i \phi}\right) \neq 0\right\} .
$$

By straightforward computations, it can be seen that $T_{u, v}=\mathbb{R}$ and so $V_{J}(A)$ has not a support line in the direction $\theta$. If the eigenvalues of $J A^{\prime}$ are not all simple, by a perturbation we can ensure that they become all simple and the result follows by a continuity argument.

We observe that the converse of Proposition 2.1 does not hold, in general, as shown in Example 3 (Section 8). However, the converse is true in the $2 \times 2$ case, as will be seen in the proof of Theorem 3.2.

A boundary point $\mu$ of a subset $K$ in $\mathbb{C}$ is a corner of $K$ if there exists a sufficiently small $\epsilon>0$ such that the intersection of $K$ and the circular disc $\mathcal{D}=\{v \in \mathbb{C}:|v-\mu|<\epsilon\}$ is contained in a sector of $\mathcal{D}$ of degree less than $\pi$.

The connection between the corners of $V_{S}(A)$ and the eigenvalues of $S^{-1} A$ is described in the next result, which will be used in the proof of Theorem 2.2 .

Theorem 2.1. (Li and Rodman [14]) Let $A \in M_{n}$. If $z \in V_{S}(A)$ is a corner of $V_{S}(A)$, then $z$ is an eigenvalue of $S^{-1} A$ and there exists an eigenvector $x$ associated to $z$, such that $A x=z S x, A^{*} x=\bar{z} S x$ and $x^{*} S x= \pm 1$.

Murnaghan [16] and Kippenhahn [11], independently, showed that the boundary of the classical numerical range of a matrix $A \in M_{n}$ is the set of real points of the algebraic curve (of class $n$ ) with equation in line coordinates

$$
\operatorname{det}\left(u H+v G+w I_{n}\right)=0
$$

where $H$ and $G$ are Hermitian matrices satisfying $A=H+i G$. The real part of this algebraic curve is denoted by $C(A)$ and the $n$ real foci of $C(A)$ are the eigenvalues of $A$.

Now, we obtain a generalization of this result for the $S$-numerical range, where $S$ is a nonsingular Hermitian matrix. 
Theorem 2.2. Let $u x+v y+w=0$ be the equation of a support line of $V_{S}(A)$ and let $A=H+i G$, where $H$ and $G$ are Hermitian matrices. Then

$$
\operatorname{det}(u H+v G+w S)=0 .
$$

Proof. For $A \in M_{n}$ and $\varphi \in \mathbb{R}, \operatorname{Re}\left(e^{-i \varphi} A\right)$ is a Hermitian matrix and its $S$-numerical range is the projection of $V_{S}\left(e^{-i \varphi} A\right)$ on the real line.

Consider the set $\Omega$ of angles $\varphi \in \mathbb{R}$ for which $V_{S}\left(\operatorname{Re}\left(e^{-i \varphi} A\right)\right)$ is either a line segment or the union of two disjoint closed half lines and denote their endpoints by $z_{j}\left(e^{-i \varphi} A\right), j=1,2$. Clearly,

$$
x=z_{j}\left(e^{-i \varphi} A\right), \quad j=1,2,
$$

are support lines of $V_{S}\left(e^{-i \varphi} A\right)$. Performing a rotation of angle $\varphi$ on the lines (4), we find that

$$
\cos \varphi x+\sin \varphi y=z_{j}\left(e^{-i \varphi} A\right), \quad j=1,2,
$$

are support lines of $e^{i \varphi} V_{S}\left(e^{-i \varphi} A\right)=V_{S}(A)$. As $\varphi$ varies in $\Omega$, all the support lines of the $S$-numerical range of $A$ are obtained. That is, given the support line of $V_{S}(A)$ of equation $u x+v y+w=0$, there exist an angle $\varphi_{0} \in \Omega$, a non-zero real scalar $\lambda$ and $j=1,2$ such that

$$
u=\lambda \cos \varphi_{0}, \quad v=\lambda \sin \varphi_{0} \quad \text { and } \quad w=-\lambda z_{j}\left(e^{-i \varphi_{0}} A\right) .
$$

For $A=H+i G$, where $H$ and $G$ are Hermitian matrices, we have

$$
S^{-1} \operatorname{Re}\left(e^{-i \varphi} A\right)=\cos \varphi S^{-1} H+\sin \varphi S^{-1} G .
$$

If $\mu$ is an eigenvalue of this matrix, then it satisfies the equation

$$
\operatorname{det}\left(\cos \varphi S^{-1} H+\sin \varphi S^{-1} G-\mu I_{n}\right)=0 .
$$

Taking into account that $\operatorname{det} S \neq 0$, we get

$$
\operatorname{det}(\cos \varphi H+\sin \varphi G-\mu S)=0 .
$$

Since $z_{j}\left(e^{-i \varphi} A\right)$ is a corner of $V_{S}\left(\operatorname{Re}\left(e^{-i \varphi} A\right)\right)$, by Theorem 2.1, it is an eigenvalue of the matrix $S^{-1} \operatorname{Re}\left(e^{-i \varphi} A\right)$ and therefore it satisfies (6). Taking $\varphi=\varphi_{0}$ and recalling (5), we obtain

$$
\operatorname{det}(u H+v G+w S)=0 .
$$

Remark 1) If the eigenvalues of $S^{-1} \operatorname{Re}\left(e^{-i \varphi} A\right)$ are all real only if $\varphi=$ $\varphi_{0}+n \pi, n \in \mathbb{Z}, V_{S}(A)$ is the whole complex plane, the complex plane except the line (or a subset of the line) $\cos \varphi_{0} x+\sin \varphi_{0} y=z_{\varphi_{0}}$, where $z_{\varphi_{0}}$ is the minimum or the maximum of the eigenvalues of $S^{-1} \operatorname{Re}\left(e^{-i \varphi_{0}} A\right)$. 
2) Since $\operatorname{det}(u H+v G+w S)$ is a homogeneous polynomial of degree $n$, we showed that the equation

$$
\operatorname{det}(u H+v G+w S)=0
$$

with $u, v, w$ viewed as homogeneous line coordinates, defines an algebraic curve of class $n$, whose real part, in the sequel denoted by $C_{S}(A)$, forms the boundary of $V_{S}(A)$. This curve has class $n$ and has real coefficients, thus it has $n$ real foci $[18,19]$, corresponding to the eigenvalues of the matrix $S^{-1} A$.

3) The dual curve of a conic is again a conic. Hence, if $A$ is a $2 \times 2$ matrix, the equation (7) defines a conic and so its real part is a hyperbola, a parabola or an ellipse, possibly degenerate. If $A$ is a $S$-scalar matrix, then by property $\mathrm{P} 5, V_{S}(A)$ is a singleton. Otherwise, if $S$ is nonsingular indefinite, then $V_{S}(A)$ is unbounded and $p$-convex, hence $C_{S}(A)$ must be an hyperbola, whose foci are the eigenvalues of $S^{-1} A$. Obviously, $V_{S}(A)$ consists of the hyperbola and its interior. If $S$ is definite, then $V_{S}(A)$ is bounded and convex, therefore the convex hull of $C_{S}(A)$ is an elliptical disc, with the eigenvalues of $S^{-1} A$ as foci.

4) If the inertia matrix of $S$ is $I_{n}$, Theorem 2.2 reduces to the MurnaghanKippenhahn theorem.

Using a theorem of Tarski, it was shown in [4] that the boundaries of $W_{C}(A)$ and $\triangle_{C}(A)$ are finite unions of algebraic arcs (see also [17]). We now apply this result to prove that the boundaries of $V_{S, C}(A)$ and $D_{S, C}(A)$ are also finite unions of algebraic arcs.

Consider $\mathbb{Z}\left[t_{1}, \ldots, t_{r} ; x_{1}, \ldots, x_{s}\right]$ the polynomial ring over the integers of the polynomials $f\left(t_{1}, \ldots, t_{r} ; x_{1}, \ldots, x_{s}\right)$ in the variables $x_{1}, \ldots, x_{s}$ and with coefficients in $\mathbb{Z}\left[t_{1}, \ldots, t_{r}\right]$. Tarski proved the following theorem [9]:

Theorem 2.3. (Tarski Theorem) Let $f_{1}, \ldots, f_{m}$ and $g_{1}, \ldots, g_{n}$ be polynomials in $\mathbb{Z}\left[t_{1}, \ldots, t_{r} ; x_{1}, \ldots, x_{s}\right]$. It is possible to find, in a finite number of steps, a finite collection $\psi_{1}, \ldots, \psi_{p}$, where each $\psi_{l}$ is a set of polynomials

$$
\psi_{l}=\left\{F_{l_{1}}, \ldots, F_{l_{p_{l}}} ; G_{l_{1}}, \ldots, G_{l_{q_{l}}}\right\}
$$


with $F_{l_{i}}, G_{l_{j}} \in \mathbb{Z}\left[t_{1}, \ldots, t_{r}\right], 1 \leq i \leq p_{l}, 1 \leq j \leq q_{l}$, such that for any real closed field $R$ and $\left(c_{1}, \ldots, c_{r}\right) \in R$,

$$
\begin{aligned}
& f_{1}\left(c_{1}, \ldots, c_{r} ; x_{1}, \ldots, x_{s}\right)=0, \ldots, f_{m}\left(c_{1}, \ldots, c_{r} ; x_{1}, \ldots, x_{s}\right)=0, \\
& g_{1}\left(c_{1}, \ldots, c_{r} ; x_{1}, \ldots, x_{s}\right)>0, \ldots, g_{n}\left(c_{1}, \ldots, c_{r} ; x_{1}, \ldots, x_{s}\right)>0
\end{aligned}
$$

have a solution if and only if there exists at least one $l, 1 \leq l \leq p$, such that

$$
F_{l_{i}}\left(c_{1}, \ldots, c_{r}\right)=0, \quad 1 \leq i \leq p_{l}, \quad G_{l_{j}}\left(c_{1}, \ldots, c_{r}\right)>0, \quad 1 \leq j \leq q_{l},
$$

also have a solution.

Theorem 2.4. Let $c=\left(\gamma_{1}, \ldots, \gamma_{n}\right) \in \mathbb{C}^{n}$ and $A=\operatorname{diag}\left(\alpha_{1}, \ldots, \alpha_{n}\right) \in M_{n}$. The boundary of $V_{S, c}(A)$ is a finite union of algebraic arcs and so a curve of class $\mathbb{C}^{\infty}$, except for a finite number of points.

Proof: Firstly, we show that the conditions of Tarski theorem are satisfied. By definition, $z \in V_{S, c}(A)$ if and only if there exists $U \in M_{n}$ such that $U^{*} S U=S$ and

$$
z=\operatorname{Tr}\left(\operatorname{diag}\left(\gamma_{1}, \ldots, \gamma_{n}\right) U^{*} \operatorname{diag}\left(\alpha_{1}, \ldots, \alpha_{n}\right) U\right) .
$$

Let $U=\left[x_{k j}+i y_{k j}\right], S=\left[a_{k j}+i b_{k j}\right]$, and $z=z_{1}+i z_{2}$, where $x_{k j}, y_{k j}, a_{k j}, b_{k j}$, $z_{1}, z_{2} \in \mathbb{R}$. The points of $V_{S, c}(A)$ satisfy the following conditions on the variables $x_{k j}$ and $y_{k j}$ :

$$
\begin{aligned}
& z_{1}+i z_{2}-\operatorname{Tr}\left(\operatorname{diag}\left(\gamma_{1}, \ldots, \gamma_{n}\right)\left[x_{k j}+i y_{k j}\right]^{*} \operatorname{diag}\left(\alpha_{1}, \ldots, \alpha_{n}\right)\left[x_{k j}+i y_{k j}\right]\right)=0, \\
& \sum_{k, l=1}^{n}\left(x_{k m}-i y_{k m}\right)\left(a_{k l}+i b_{k l}\right)\left(x_{l r}+i y_{l r}\right)-\left(a_{m r}+i b_{m r}\right)=0, \quad 1 \leq m, r \leq n .
\end{aligned}
$$

The left hand sides of these equations are polynomials on the parameters $\operatorname{Re} \alpha_{h}, \operatorname{Re} \gamma_{h}, \operatorname{Im} \alpha_{h}, \operatorname{Im} \gamma_{h}, z_{1}, z_{2}, a_{k j}, b_{k j}$ and on the variables $x_{k j}$ and $y_{k j}$. Considering the real and the imaginary parts of the left hand sides of the previous equations and equating them to 0 , we obtain a set of polynomial equations with integer coefficients on the same parameters and variables. Let

$$
w=\left(\left(\operatorname{Re} \alpha_{h}, \operatorname{Re} \gamma_{h}, \operatorname{Im} \alpha_{h}, \operatorname{Im} \gamma_{h}, h=1, \ldots, n\right),\left(a_{k j}, b_{k j}, k, j=1, \ldots, n\right),\left(z_{1}, z_{2}\right)\right) .
$$

Due to Tarski Theorem, we can conclude that there exists a finite collection $\psi_{1}, \ldots, \psi_{p}$, where each $\psi_{l}$ is a set of polynomials

$$
\psi_{l}=\left\{F_{l_{1}}, \ldots, F_{l_{n_{l}}} ; G_{l_{1}}, \ldots, G_{l_{m_{l}}}\right\}
$$


with $F_{l_{s}}, G_{l_{q}} \in \mathbb{Z}\left[t_{1}, \ldots, t_{n^{2}+4 n+2}\right], 1 \leq s \leq n_{l}, 1 \leq q \leq m_{l}$, such that for all $\alpha_{h}, \gamma_{h}, a_{k j}+i b_{k j}, x_{k j}+i y_{k j}, z \in \mathbb{C}$, the initial equations have a solution if and only if there exists at least one $l, 1 \leq l \leq p$, such that

$$
F_{l_{s}}(w)=0, \quad 1 \leq s \leq n_{l}, \quad G_{l_{q}}(w)>0, \quad 1 \leq q \leq m_{l},
$$

have a solution. A point for which only the inequalities in (8) are satisfied (and no equality) does not belong to the boundary of $V_{S, c}(A)$. The boundary is formed by a finite number of algebraic arcs. The boundary points are of class $\mathbb{C}^{\infty}$, unless they satisfy equations (8), for at least two values of l. (Observe that the number of points for which this happens is finite $[9$, p.325].)

In an analogous way, the following result can be obtained for the $S, c$ determinantal range.

Theorem 2.5. Let $c=\left(\gamma_{1}, \ldots, \gamma_{n}\right) \in \mathbb{C}^{n}$ and $A=\operatorname{diag}\left(\alpha_{1}, \ldots, \alpha_{n}\right) \in M_{n}$. The boundary of $D_{S, c}(A)$ is a finite union of algebraic arcs and so it is a curve of class $\mathbb{C}^{\infty}$, except for a finite number of points.

Notice that the previous results can be easily generalized for arbitrary matrices $A \in M_{n}$. They are also generalizable for $V_{S, C}(A)$ and $D_{S, C}(A)$, where $A, C \in M_{n}$.

\section{The Hyperbolical Range Theorem}

In the theory of classical numerical ranges and its generalizations, the reduction of problems to its bidimensional case is a very useful technique. For instance, convexity results can be proved using such a reduction. In this theory, the elliptical range theorem is a particularly important result. It asserts that the classical numerical range of a $2 \times 2$ matrix $A$ is an elliptical disc, possibly degenerate, with the eigenvalues $\lambda_{1}$ and $\lambda_{2}$ of $A$ as foci and minor axis of length

$$
\sqrt{\operatorname{Tr}\left(A^{*} A\right)-\left|\lambda_{1}\right|^{2}-\left|\lambda_{2}\right|^{2}}
$$

In this section, we consider $J=I_{r} \oplus-I_{2-r}$, with $0 \leq r \leq 2$, and give a detailed geometric description of the $J$-numerical range of $A$ (see [13] for an alternative approach). Only $r=1$ is considered, since for $r=0$ and $r=2, V_{J}(A)= \pm W(A)$ is characterized by the elliptical range theorem. In particular, for $r=1$, a hyperbolical range theorem is explicitly obtained. In the degenerate cases, $V_{J}(A)$ is a subset of a line, the whole complex plane or the complex plane except a line. 
Firstly, we prove the hyperbolical range theorem for a certain class of matrices.

Theorem 3.1. Let

$$
M_{1}=\left[\begin{array}{cc}
1 & a e^{i \theta} \\
b e^{i \theta} & 1
\end{array}\right],
$$

with $a, b \geq 0, a \neq b$ and $\theta \in \mathbb{R}$, and let

$$
\beta=2 \sqrt{1-2 a b \cos (2 \theta)+a^{2} b^{2}}+2-a^{2}-b^{2} .
$$

(i) If $\beta<0$, then $V_{J}\left(M_{1}\right)$ is the whole complex plane.

(ii) If $\beta=0$, then $V_{J}\left(M_{1}\right)$ is the whole complex plane except a line.

(iii) If $\beta>0$, then $V_{J}\left(M_{1}\right)$ is bounded by a hyperbola centered at the origin with transverse axis of length $\sqrt{\beta}$.

Proof: For $\varphi \in \mathbb{R}$, consider the matrix $J \operatorname{Re}\left(e^{i \varphi} M_{1}\right)$. The eigenvalues of this matrix are

$$
\lambda_{i}(\varphi)= \pm \frac{1}{2} \sqrt{2-a^{2}-b^{2}+2 \cos (2 \varphi)-2 a b \cos (2 \theta+2 \varphi)}, \quad i=1,2,
$$

and the eigenvectors associated with $\lambda_{i}(\varphi)$ are given by

$$
u_{i}(\varphi)=\left(-2 \cos \varphi-2 \lambda_{i}(\varphi),(a+b) \cos (\theta+\varphi)-i(a-b) \sin (\theta+\varphi)\right) .
$$

These eigenvectors satisfy the following relation

$u_{i}(\varphi)^{*} J u_{i}(\varphi)=4\left(\overline{\lambda_{i}(\varphi)}+\cos \varphi\right)\left(\lambda_{i}(\varphi)+\cos \varphi\right)-a^{2}-b^{2}-2 a b \cos (2 \theta+2 \varphi)$, $i=1,2$.

Observe that $1-2 a b \cos (2 \theta)+a^{2} b^{2}=0$ if and only if $a b=1$ and $\theta=$ $n \pi, n \in \mathbb{Z}$. Then $a \neq 1$ (otherwise $a=b$, contradicting the hypothesis). Hence

$$
\beta=-\frac{\left(1-a^{2}\right)^{2}}{a^{2}}<0 \quad \text { and } \quad \lambda_{i}(\varphi)= \pm \frac{\sqrt{\beta}}{2}, \quad i=1,2,
$$

for all $\varphi \in \mathbb{R}$, that is, the eigenvalues of $J \operatorname{Re}\left(e^{i \varphi} M_{1}\right)$ are pure imaginary complex numbers. Thus, there does not exist any support line of $V_{J}\left(M_{1}\right)$ in any direction and so the $J$-numerical range of $M_{1}$ is the whole complex plane.

Now, suppose that $a b \neq 1$ or $\theta \neq n \pi, n \in \mathbb{Z}$. Consider the function $\mu: \mathbb{R} \rightarrow \mathbb{R}$ defined by

$$
\mu(\varphi)=2-a^{2}-b^{2}+2 \cos (2 \varphi)-2 a b \cos (2 \theta+2 \varphi) .
$$


We easily check that

$$
\begin{aligned}
\mu(\varphi) & =2-a^{2}-b^{2}+2\left|1-a b e^{2 i \theta}\right| \cos (2 \varphi+\gamma) \\
& \leq 2-a^{2}-b^{2}+2\left|1-a b e^{2 i \theta}\right|=\beta, \quad \varphi \in \mathbb{R},
\end{aligned}
$$

where

$$
\tan \gamma=\frac{a b \sin (2 \theta)}{a b \cos (2 \theta)-1}
$$

Therefore, $\beta$ is the maximum of $\mu$ and is attained at $\varphi_{0}=k \pi-\gamma / 2, k \in \mathbb{Z}$.

We prove (i). If $\beta<0$, it is clear that $\mu(\varphi)<0$. It follows that

$$
\lambda_{i}(\varphi)= \pm \frac{1}{2} \sqrt{\mu(\varphi)}, i=1,2
$$

are pure imaginary complex numbers, for all $\varphi \in \mathbb{R}$. Hence $V_{J}\left(M_{1}\right)=\mathbb{C}$.

Now, we prove (ii). If $\beta=0$, then $\mu(\varphi)<0$, for all $\varphi \in \mathbb{R}$ different from $\varphi_{0}$. Recalling that $\mu\left(\varphi_{0}\right)=0$, we find that $\lambda_{1}\left(\varphi_{0}\right)=\lambda_{2}\left(\varphi_{0}\right)=0$. The corresponding eigenvectors satisfy the condition

$$
u_{i}\left(\varphi_{0}\right)^{*} J u_{i}\left(\varphi_{0}\right)=0, \quad i=1,2 .
$$

For $\varphi \in \mathbb{R}$ different from $\varphi_{0}$, the eigenvalues of $J \operatorname{Re}\left(e^{i \varphi} M_{1}\right)$ are pure imaginary complex numbers and so $V_{J}\left(M_{1}\right)$ is the whole complex plane except the line $\cos \varphi_{0} x+\sin \varphi_{0} y=0$.

Finally, we prove (iii). If $\beta>0$, then $\mu(\varphi)>0$, for any $\varphi$ in an interval containing $\varphi_{0}$, say $(\xi, \eta)$, with $\mu(\xi)=\mu(\eta)=0$. In that interval, since $a \neq b$, we have

$$
0<\mu(\varphi) \leq 4 \cos ^{2} \varphi-(a-b)^{2}<4 \cos ^{2} \varphi
$$

and the eigenvalues of $J \operatorname{Re}\left(e^{i \varphi} M_{1}\right)$ are non-zero real numbers. It can be easily checked that

$u_{i}^{*}(\varphi) M_{1} u_{i}(\varphi)=-2\left(\lambda_{i}(\varphi)+\cos \varphi\right)\left(e^{i \theta}(a+b)^{2} \cos (\theta+\varphi)-i e^{i \theta}(a-b)^{2} \sin (\theta+\varphi)-4 \cos \varphi\right)$

and

$$
u_{i}(\varphi)^{*} J u_{i}(\varphi)=8 \lambda_{i}(\varphi)\left(\lambda_{i}(\varphi)+\cos \varphi\right) \neq 0, \quad i=1,2 .
$$

Let

$$
x+i y=\frac{u_{i}^{*}(\varphi) M_{1} u_{i}(\varphi)}{u_{i}^{*}(\varphi) J u_{i}(\varphi)}
$$


where

$$
\begin{array}{r}
x=\frac{-1}{4 \lambda_{i}(\varphi)}\left((a+b)^{2} \cos (\theta+\varphi) \cos \theta+(a-b)^{2} \sin (\theta+\varphi) \sin \theta-4 \cos \varphi\right), \\
y=\frac{-1}{4 \lambda_{i}(\varphi)}\left((a+b)^{2} \cos (\theta+\varphi) \sin \theta-(a-b)^{2} \sin (\theta+\varphi) \cos \theta\right)
\end{array}
$$

Obviously, $(x, y)$ belongs to the boundary of $V_{J}\left(M_{1}\right)$.

After some computations, from (9) and (10), we get

$$
\left\{\begin{array}{l}
A_{1} \cos (2 \varphi)+B_{1} \sin (2 \varphi)=C_{1} \\
A_{2} \cos (2 \varphi)+B_{2} \sin (2 \varphi)=C_{2}
\end{array}\right.
$$

where

$$
\begin{array}{lll}
A_{1}=C^{2}-B^{2}-8 D x^{2}, & B_{1}=2 B\left(C+4 x^{2}\right), & C_{1}=8 E x^{2}-B^{2}-C^{2}, \\
A_{2}=B^{2}-A^{2}-8 D y^{2}, & B_{2}=2 B\left(A+4 y^{2}\right), & C_{2}=8 E y^{2}-A^{2}-B^{2}
\end{array}
$$

and

$$
\begin{aligned}
& A=a^{2}+b^{2}-2 a b \cos (2 \theta), \\
& B=-2 a b \sin (2 \theta), \\
& C=a^{2}+b^{2}+2 a b \cos (2 \theta)-4, \\
& D=2-2 a b \cos (2 \theta), \\
& E=2-a^{2}-b^{2} .
\end{aligned}
$$

Eliminating $\varphi$ in (11), we get the quadratic equation

$$
A x^{2}+2 B x y+C y^{2}+F=0,
$$

with $F=\left(a^{2}-b^{2}\right)^{2} / 4-A$. Reducing the conic in (12) to its main axis, we get

$$
\frac{X^{2}}{\beta}-\frac{Y^{2}}{\alpha}=\frac{1}{4}
$$

where

$$
\alpha=2 \sqrt{1-2 a b \cos (2 \theta)+a^{2} b^{2}}-2+a^{2}+b^{2} .
$$

It can be easily seen that $\alpha$ is nonnegative. Moreover, $\alpha=0$ if and only if either $a=b=0$ or $a=b \leq 1$ and $\theta=n \pi, n \in \mathbb{Z}$. In this case, $\alpha>0$. Hence, the equation (13) describing the boundary of $V_{J}\left(M_{1}\right)$ is a hyperbola centered at the origin, with transverse axis of length $\sqrt{\beta}$ and non-transverse axis of length $\sqrt{\alpha}$. 
We observe that the complete characterization of the hyperbola in Theorem 3.1 (iii) is discussed in Theorem 3.2.

The following two lemmas are used in the proof of Theorem 3.2.

Lemma 3.1. Let $a, b \geq 0, a \neq b$. The envelope of the family of curves over the parameter $r \in \mathbb{R}, r^{2} \geq 1$,

$$
\frac{((x-r) \sin \theta-y \cos \theta)^{2}}{(a-b)^{2}}+\frac{((x-r) \cos \theta+y \sin \theta)^{2}}{(a+b)^{2}}=\frac{r^{2}-1}{4}
$$

in an adequate coordinate system is given by

$$
\alpha X^{2}-\beta Y^{2}=\frac{\alpha \beta}{4}
$$

where

$$
\begin{aligned}
& \alpha=2 \sqrt{1-2 a b \cos (2 \theta)+a^{2} b^{2}}-2+a^{2}+b^{2}, \\
& \beta=2 \sqrt{1-2 a b \cos (2 \theta)+a^{2} b^{2}}+2-a^{2}-b^{2} .
\end{aligned}
$$

Proof: Evaluating the envelope of the family of curves (14), we obtain

$$
A x^{2}+2 B x y+C y^{2}+F=0,
$$

where

$$
\begin{aligned}
& A=a^{2}+b^{2}-2 a b \cos (2 \theta), \\
& B=-2 a b \sin (2 \theta), \\
& C=a^{2}+b^{2}+2 a b \cos (2 \theta)-4, \\
& F=\left(a^{2}-b^{2}\right)^{2} / 4-A .
\end{aligned}
$$

Reducing the conic in (15) to its main axis, we get

$$
\alpha X^{2}-\beta Y^{2}=\frac{\alpha \beta}{4}
$$

where $\alpha$ and $-\beta$ are the eigenvalues of the real symmetric matrix associated with the quadratic form $A x^{2}+2 B x y+C y^{2}$,

$$
S=\left[\begin{array}{ll}
A & B \\
B & C
\end{array}\right]
$$

It is now straightforward to complete the proof. 
Lemma 3.2. For $A=\left(a_{i j}\right) \in M_{2}$, there exists a pseudo-unitary matrix $U$ such that

$$
U^{*} A U=\left[\begin{array}{cc}
a_{11} & \left|a_{12}\right| e^{i \vartheta} \\
\left|a_{21}\right| e^{i \vartheta} & a_{22}
\end{array}\right],
$$

where $2 \vartheta$ is the sum of the arguments of $a_{12}$ and $a_{21}$.

Proof: The diagonal matrix $U=\operatorname{diag}\left(e^{i \eta}, e^{i \mu}\right)$, such that $2(\eta-\mu)=\arg a_{12}-$ $\arg a_{21}$, is pseudo-unitary and satisfies the asserted property.

Theorem 3.2. (Hyperbolical Range Theorem) The J-numerical range of a matrix $A \in M_{2}$ is bounded by a (possibly degenerate) hyperbola, with the eigenvalues $\lambda_{1}$ and $\lambda_{2}$ of $J A$ as foci and with non-transverse axis of length

$$
\sqrt{\left|\lambda_{1}\right|^{2}+\left|\lambda_{2}\right|^{2}-\operatorname{Tr}\left(A^{*} J A J\right)}
$$

For the degenerate cases, $V_{J}(A)$ is a singleton, a line, a subset of a line, the whole complex plane, or the complex plane except a line.

Proof: Let $A=\left(a_{i j}\right)$ be a zero trace matrix in $M_{2}$. By Lemma 3.2, there exists a pseudo-unitary matrix $U$ such that

$$
U^{*} A U=\frac{1}{2} \operatorname{Tr}(A J) J+\left[\begin{array}{cc}
0 & a e^{i \theta} \\
b e^{i \theta} & 0
\end{array}\right]
$$

where $a$ and $b$ are the moduli of $a_{12}$ and $a_{21}$, respectively, and $2 \theta=\arg a_{12}+$ $\arg a_{21}$.

Now, let $A=\left(a_{i j}\right)$ be a non-zero trace matrix in $M_{2}$. By Lemma 3.2, there exists a pseudo-unitary matrix $U$ such that

$$
\frac{2}{\operatorname{Tr}(A)} U^{*} A U-\frac{\operatorname{Tr}(A J)}{\operatorname{Tr}(A)} J=\left[\begin{array}{cc}
1 & a e^{i \theta} \\
b e^{i \theta} & 1
\end{array}\right],
$$

where $a$ and $b$ are the moduli of $2 a_{12} / \operatorname{Tr}(A)$ and $2 a_{21} / \operatorname{Tr}(A)$, respectively, and

$$
2 \theta=\arg a_{12}+\arg a_{21}-2 \arg \operatorname{Tr}(A) .
$$

Without loss of generality, we may concentrate on the study of the $J$ numerical range of the matrices

$$
M_{k}=\left[\begin{array}{cc}
k & a e^{i \theta} \\
b e^{i \theta} & k
\end{array}\right], \quad k=0,1 .
$$

The eigenvalues of $J M_{k}$ are of the form $\pm \sqrt{k^{2}-a b e^{2 i \theta}}$. 
Using properties $\mathrm{P} 1$ and $\mathrm{P} 2$, the $J$-numerical range of the matrix $A$ can be easily obtained from

$V_{J}\left(M_{k}\right)=\left\{\frac{k\left|x_{1}\right|^{2}+k\left|x_{2}\right|^{2}+\left(a \overline{x_{1}} x_{2}+b x_{1} \overline{x_{2}}\right) e^{i \theta}}{\left|x_{1}\right|^{2}-\left|x_{2}\right|^{2}}: x_{1}, x_{2} \in \mathbb{C},\left|x_{1}\right| \neq\left|x_{2}\right|\right\}$,

where $k=0$, if $A$ is a zero trace matrix and $k=1$, otherwise. Taking

$$
r=\frac{\left|x_{1}\right|^{2}+\left|x_{2}\right|^{2}}{\left|x_{1}\right|^{2}-\left|x_{2}\right|^{2}}, \quad \quad \phi=\arg x_{2}-\arg x_{1},
$$

(19) can be written in the form

$$
V_{J}\left(M_{k}\right)=\left\{r k+\frac{1}{2} \sqrt{r^{2}-1}\left(a e^{i \phi}+b e^{-i \phi}\right) e^{i \theta}: r^{2}-1 \geq 0, \phi \in \mathbb{R}\right\} .
$$

Consider, firstly, $k=0$. If $a=b=0$, then clearly $V_{J}\left(M_{0}\right)=\{0\}$. If $a=b \neq 0$, the eigenvalues of $J M_{0}$ are $\pm a i e^{i \theta}$ and so $V_{J}\left(M_{0}\right)=e^{i \theta} \mathbb{R}$, the line passing through 0 and perpendicular to the line segment joining these eigenvalues. For $a \neq b, e^{i \theta}(x+i y) \in V_{J}\left(M_{0}\right)$ if and only if

$$
\frac{x^{2}}{(a+b)^{2}}+\frac{y^{2}}{(a-b)^{2}}=\frac{r^{2}-1}{4}
$$

with $r^{2}-1 \geq 0$, and so $V_{J}\left(M_{0}\right)=\mathbb{C}$.

Now, let $k=1$. Two cases are possible: I) $a \neq b$; II) $a=b$.

I) Clearly $x+i y \in V_{J}\left(M_{1}\right)$ if and only if

$$
\begin{aligned}
& x=r+\frac{1}{2} \sqrt{r^{2}-1}((a+b) \cos \theta \cos \phi-(a-b) \sin \theta \sin \phi), \\
& y=\frac{1}{2} \sqrt{r^{2}-1}((a+b) \sin \theta \cos \phi+(a-b) \cos \theta \sin \phi) .
\end{aligned}
$$

Since $a \neq b$, after some computations, we obtain

$$
\frac{((x-r) \sin \theta-y \cos \theta)^{2}}{(a-b)^{2}}+\frac{((x-r) \cos \theta+y \sin \theta)^{2}}{(a+b)^{2}}=\frac{r^{2}-1}{4},
$$

where the parameter $r$ is such that $r^{2}-1 \geq 0$. By Lemma 3.1, the envelope of this family of curves is given by

$$
\alpha X^{2}-\beta Y^{2}=\frac{\alpha \beta}{4}
$$


where

$$
\begin{aligned}
& \alpha=2 \sqrt{1-2 a b \cos (2 \theta)+a^{2} b^{2}}-2+a^{2}+b^{2}, \\
& \beta=2 \sqrt{1-2 a b \cos (2 \theta)+a^{2} b^{2}}+2-a^{2}-b^{2} .
\end{aligned}
$$

As already seen in the proof of Theorem 3.1 (iii), since $a \neq b, \alpha$ is positive.

Obviously,

$$
\operatorname{Tr}\left(M_{1} J M_{1}^{*} J\right)=2-a^{2}-b^{2} .
$$

Since the eigenvalues $\lambda_{1}^{\prime}$ and $\lambda_{2}^{\prime}$ of $J M_{1}$ are of the form $\pm \sqrt{1-a b e^{2 i \theta}}$, we have

$$
\begin{aligned}
& \alpha=\left|\lambda_{1}^{\prime}\right|^{2}+\left|\lambda_{2}^{\prime}\right|^{2}-\operatorname{Tr}\left(M_{1} J M_{1}^{*} J\right), \\
& \beta=\left|\lambda_{1}^{\prime}\right|^{2}+\left|\lambda_{2}^{\prime}\right|^{2}+\operatorname{Tr}\left(M_{1} J M_{1}^{*} J\right) .
\end{aligned}
$$

The eigenvectors of the matrix $S$ in (16), associated with $\alpha$, have the direction of the vector in $\mathbb{R}^{2}$

$$
u=\left(\left|\lambda_{1}^{\prime}\right|^{2}-1+a b \cos (2 \theta), a b \sin (2 \theta)\right) .
$$

Identifying vectors in $\mathbb{R}^{2}$ with complex numbers, the following condition for complexes is satisfied

$$
u^{2}=2\left(1-a b \cos (2 \theta)-\left|\lambda_{1}^{\prime}\right|^{2}\right) \lambda_{1}^{\prime 2} .
$$

Thus, the line defined by the eigenvalues $\lambda_{1}^{\prime}$ and $\lambda_{2}^{\prime}$ has the direction of the vector $u$. We now classify the conic describing the boundary of $V_{J}\left(M_{1}\right)$, according to the sign of $\alpha \beta$, which depends only on the sign of $\beta$, due to the positivity of $\alpha$.

i) If $\beta>0,(20)$ is just

$$
\frac{X^{2}}{\beta}-\frac{Y^{2}}{\alpha}=\frac{1}{4},
$$

the equation of a hyperbola centered at the origin, with transverse and nontransverse axis of length $\sqrt{\beta}$ and $\sqrt{\alpha}$, respectively, and semi-focal distance given by $\left|\lambda_{1}^{\prime}\right|=\left|\lambda_{2}^{\prime}\right|$. The direction of the transverse axis of the hyperbola is that of the vector $u$. This means that the foci of the hyperbola are precisely the eigenvalues $\lambda_{1}^{\prime}$ and $\lambda_{2}^{\prime}$ of $J M_{1}$.

ii) If $\beta=0$, then equation (20) reduces to $X=0$, that is, the line through the origin perpendicular to the line segment defined by the eigenvalues $\lambda_{1}^{\prime}$ and $\lambda_{2}^{\prime}$ of the matrix $J M_{1}$. By Theorem 3.1 (ii), $V_{J}\left(M_{1}\right)$ is the whole complex 
plane except this line. (This line is the boundary of the open half plane representing $V_{J}^{+}\left(M_{1}\right)$.)

iii) If $\beta<0$, due to Theorem 3.1 (i), $V_{J}\left(M_{1}\right)=\mathbb{C}$.

This finishes the proof of I).

II) If $a=b=0$, then obviously $\left.V_{J}\left(M_{1}\right)=\mathbb{R} \backslash\right]-1,1[$. Now, let $a=b \neq 0$.

If $\theta=n \pi, n \in \mathbb{Z}$, it is clear that $V_{J}\left(M_{1}\right)$ is a subset of the real line. We observe that the elements in $V_{J}^{+}\left(M_{1}\right)\left(-V_{J}^{-}\left(M_{1}\right)\right)$ are given by the ranges of the family of functions $f_{\phi}:[1,+\infty) \rightarrow \mathbb{R}\left(f_{\phi}:(-\infty,-1] \rightarrow \mathbb{R}\right)$ defined by

$$
f_{\phi}(r)=r+\sqrt{r^{2}-1} \cos \phi a, \quad \phi \in \mathbb{R} .
$$

We observe that $f_{0}(-r)=-f_{\pi}(r)$ and $f_{\pi}(-r)=-f_{0}(r)$. Since $f_{\pi} \leq f_{\phi} \leq f_{0}$, we just evaluate the extreme values of the functions $f_{\pi}$ and $f_{0}$. For $a<1$, we conclude that $\sqrt{1-a^{2}}$ is the minimum of $\left.f_{\pi}\right|_{[1,+\infty)}$, while $\left.f_{0}\right|_{[1,+\infty)}$ does not have a maximum, and so $V_{J}^{+}\left(M_{1}\right)=\left[\sqrt{1-a^{2}},+\infty\right)$. Thus,

$$
\left.V_{J}\left(M_{1}\right)=\mathbb{R} \backslash\right]-\sqrt{1-a^{2}}, \sqrt{1-a^{2}}[.
$$

This is just the line defined by the eigenvalues of $J M_{1}$, except the open line segment with these eigenvalues as endpoints. For $a \geq 1$, neither $f_{\pi}$ admits minimum nor $f_{0}$ maximum. In particular, when $a=1$, we have $V_{J}\left(M_{1}\right)=\mathbb{R} \backslash\{0\}$. (In fact, if $0 \in V_{J}^{+}\left(M_{1}\right)$, there exist $r \geq 1$ and $\phi \in \mathbb{R}$ such that

$$
0=r+\sqrt{r^{2}-1} \cos \phi \geq r-\sqrt{r^{2}-1}>0,
$$

a contradiction. In an analogous way, if $0 \in-V_{J}^{-}\left(M_{1}\right)$, we get a contradiction.) If $a>1$, then $V_{J}\left(M_{1}\right)=\mathbb{R}$. (We observe that, in this case, the eigenvalues of $J M_{1}$ are pure imaginary complex numbers.)

If $\theta \neq n \pi, n \in \mathbb{Z}$, then the elements in $V_{J}\left(M_{1}\right)$ are characterized by the family of line segments contained in $y \cos \theta=(x-r) \sin \theta$, where $r^{2}-1 \geq 0$ and with endpoints at $y= \pm \sqrt{r^{2}-1} a \sin \theta$. Eliminating the parameter $r$ between these two equations, we get

$$
(x-\operatorname{cotg} \theta y)^{2}-\left(\frac{y}{a \sin \theta}\right)^{2}=1 .
$$


It is straightforward to verify that this equation describes a hyperbola, which is the equation (15) in the case $a=b$. Since $\beta>0, V_{J}\left(M_{1}\right)$ is a hyperbola like the one in I) i).

We have finished the description of the $J$-numerical range of the matrices $M_{k}$, for $k=0,1$. Now, we give a detailed description of $V_{J}(A)$. Before that, we observe that the eigenvalues $\lambda_{1}$ and $\lambda_{2}$ of $J A$ are given by

$$
\frac{1}{2} \operatorname{Tr}(A J) \pm \frac{1}{2} \sqrt{(\operatorname{Tr}(A))^{2}-4 a_{12} a_{21}} .
$$

If $A$ is a zero trace matrix, having in mind (17) and properties $\mathrm{P} 1$ and $\mathrm{P} 2$, we get

$$
V_{J}(A)=\frac{1}{2} \operatorname{Tr}(A J)+V_{J}\left(M_{0}\right) .
$$

From the above discussion, we conclude that $V_{J}(A)$ is a point, $\operatorname{Tr}(A J) / 2$, if $A$ is a $J$-scalar matrix; the line passing through $\operatorname{Tr}(A J) / 2$ and perpendicular to the line segment joining the eigenvalues of $J A$, if $\left|a_{12}\right|=\left|a_{21}\right| \neq 0$; and the whole complex plane, otherwise.

Now, if $A$ is a non-zero trace matrix, from (18) and properties P1 and P2, it follows that

$$
V_{J}(A)=\frac{1}{2} \operatorname{Tr}(A J)+\frac{1}{2} \operatorname{Tr}(A) V_{J}\left(M_{1}\right) .
$$

Let $\lambda_{1}$ and $\lambda_{2}$ be the eigenvalues of $J A$. Taking into account (18) and the fact that $U$ is pseudo-unitary, the following relation holds

$$
\lambda_{i}=\frac{1}{2} \operatorname{Tr}(A J)+\frac{1}{2} \operatorname{Tr}(A) \lambda_{i}^{\prime}, \quad i=1,2 .
$$

If $V_{J}\left(M_{1}\right)$ contains the eigenvalues $\lambda_{1}^{\prime}$ and $\lambda_{2}^{\prime}$ of $J M_{1}$, then $V_{J}(A)$ contains $\lambda_{1}$ and $\lambda_{2}$. Therefore, it follows that

$$
\frac{1}{4}|\operatorname{Tr}(A)|^{2}\left(\left|\lambda_{1}^{\prime}\right|^{2}+\left|\lambda_{2}^{\prime}\right|^{2}\right)=\left|\lambda_{1}\right|^{2}+\left|\lambda_{2}\right|^{2}-\frac{1}{2}|\operatorname{Tr}(A J)|^{2} .
$$

On the other hand, it can be easily checked that

$$
\operatorname{Tr}\left(A J A^{*} J\right)=\frac{1}{4}|\operatorname{Tr}(A)|^{2} \operatorname{Tr}\left(M_{1} J M_{1}^{*} J\right)+\frac{1}{2}|\operatorname{Tr}(A J)|^{2} .
$$

From (21) and (22), we obtain

$$
M=\frac{1}{4}|\operatorname{Tr}(A)|^{2} \alpha=\left|\lambda_{1}\right|^{2}+\left|\lambda_{2}\right|^{2}-\operatorname{Tr}\left(A J A^{*} J\right),
$$




$$
\begin{aligned}
N & =\frac{1}{4}|\operatorname{Tr}(A)|^{2} \beta=\left|\lambda_{1}\right|^{2}+\left|\lambda_{2}\right|^{2}+\operatorname{Tr}\left(A J A^{*} J\right)-|\operatorname{Tr}(A J)|^{2} \\
& =\operatorname{Tr}\left(A J A^{*} J\right)-2 \operatorname{Re}\left(\overline{\lambda_{1}} \lambda_{2}\right) .
\end{aligned}
$$

If $\left|a_{12}\right| \neq\left|a_{21}\right|$, having in mind the discussion in I), we have:

a) If $N>0, V_{J}(A)$ is bounded by a hyperbola centered at $\operatorname{Tr}(A J) / 2$, with the eigenvalues $\lambda_{1}$ and $\lambda_{2}$ of $J A$ as foci and with transverse and non-transverse axis of length $\sqrt{N}$ and $\sqrt{M}$, respectively.

b) If $N=0, V_{J}(A)$ is the complex plane, except the line passing through the point $\operatorname{Tr}(A J) / 2$ and perpendicular to the line segment joining $\lambda_{1}$ and $\lambda_{2}$. (In particular, $V_{J}^{+}(A)$ is one of the open half planes defined by that line.)

c) If $N<0, V_{J}(A)$ is the whole complex plane.

If $\left|a_{12}\right|=\left|a_{21}\right| \neq 0$, either $\arg a_{12}+\arg a_{21} \neq 2 \arg \operatorname{Tr}(A)$ and $V_{J}(A)$ is bounded by a hyperbola like the one described in a), or $\arg a_{12}+\arg a_{21}=2 \arg \operatorname{Tr}(A)$ and $V_{J}(A)$ is a subset of the line defined by

$$
a_{11}=\frac{1}{2} \operatorname{Tr}(A(J+I)) \quad \text { and } \quad-a_{22}=\frac{1}{2} \operatorname{Tr}(A(J-I)),
$$

which are the diagonal elements of $J A$. More precisely:

d) $V_{J}(A)$ is the whole line, if $2\left|a_{12}\right|>|\operatorname{Tr}(A)|$. (In this case, this line is perpendicular to the line segment joining $\lambda_{1}$ and $\lambda_{2}$ );

e) $V_{J}(A)$ is the whole line except the middle point $\operatorname{Tr}(A J) / 2$, if $2\left|a_{12}\right|=$ $|\operatorname{Tr}(A)| . \quad$ (In this case, the eigenvalues of $J A$ are just this middle point);

f) $V_{J}(A)$ is the line defined by the eigenvalues of $J A$, except the open line segment with these eigenvalues as endpoints, if $2\left|a_{12}\right|<|\operatorname{Tr}(A)|$.

Finally, if $A$ is a diagonal matrix of non-zero trace, then $V_{J}(A)$ is, as in $\mathrm{f}$ ), the line through the eigenvalues of $J A$, except the open line segment whose endpoints are these eigenvalues.

For the sake of completeness, we restate the hyperbolical range theorem for $V_{S}(A)$, when the inertia matrix of $S$ is $J=\operatorname{diag}(1,-1)$. For that purpose, recall the relation (3) between the $S$-numerical range of $A$ and the $J$-numerical range of $A_{R}=R^{*} A R$, where $R^{*} S R=J$. We observe that the eigenvalues of $J A_{R}$ coincide with the eigenvalues of $S^{-1} A$ and that $\operatorname{Tr}\left(A_{R}^{*} J A_{R} J\right)=\operatorname{Tr}\left(A^{*} S^{-1} A S^{-1}\right)$. Then $V_{S}(A)$, for $A \in M_{2}$, is bounded by a 
(possibly degenerate) hyperbola, with the eigenvalues $\lambda_{1}$ and $\lambda_{2}$ of $S^{-1} A$ as foci and with the length of non-transverse axis equal to

$$
\sqrt{\left|\lambda_{1}\right|^{2}+\left|\lambda_{2}\right|^{2}-\operatorname{Tr}\left(A^{*} S^{-1} A S^{-1}\right)}
$$

\section{4. $V_{J, c}(A)$ and $D_{J, c}(A)$ for $2 \times 2$ Matrices}

The elliptical range theorems concerning $W_{c}(A)$ and $\triangle_{c}(A)$, for $c=\left(\gamma_{1}, \gamma_{2}\right) \in$ $\mathbb{C}^{2}$ and $A \in M_{2}$, are well known (see [5] and [2]). Supposing that $\lambda_{1}$ and $\lambda_{2}$ are the eigenvalues of $A, W_{c}(A)$ is an elliptical disc (possibly degenerate) with foci $\gamma_{1} \lambda_{1}+\gamma_{2} \lambda_{2}$ and $\gamma_{1} \lambda_{2}+\gamma_{2} \lambda_{1}$, and $\triangle_{c}(A)$ is an elliptical disc (possibly degenerate) with foci $\left(\gamma_{1}+\lambda_{1}\right)\left(\gamma_{2}+\lambda_{2}\right)$ and $\left(\gamma_{1}+\lambda_{2}\right)\left(\gamma_{2}+\lambda_{1}\right)$. Both discs have minor axis of length

$$
\left|\gamma_{1}-\gamma_{2}\right| \sqrt{\operatorname{Tr}\left(A^{*} A\right)-\left|\lambda_{1}\right|^{2}-\left|\lambda_{2}\right|^{2}} .
$$

Now, we characterize the $J, c$-tracial range and the $J, c$-determinantal range of $2 \times 2$ arbitrary matrices, for $J=\operatorname{diag}(1,-1)$.

Theorem 4.1. Let $c=\left(\gamma_{1}, \gamma_{2}\right) \in \mathbb{C}^{2}, A \in M_{2}$ and let $\lambda_{1}$ and $\lambda_{2}$ be the eigenvalues of $J A$. Then

i) the $J, c$-tracial range of $A$ is bounded by a branch of a (possibly degenerate) hyperbola, with foci $\gamma_{1} \lambda_{1}-\gamma_{2} \lambda_{2}$ and $\gamma_{1} \lambda_{2}-\gamma_{2} \lambda_{1}$;

ii) the $J, c$-determinantal range of $A$ is bounded by a branch of a (possibly degenerate) hyperbola, with foci $\left(\gamma_{1}+\lambda_{1}\right)\left(\gamma_{2}-\lambda_{2}\right)$ and $\left(\gamma_{1}+\lambda_{2}\right)\left(\gamma_{2}-\lambda_{1}\right)$.

Both hyperbolas have non-transverse axis of length

$$
\left|\gamma_{1}+\gamma_{2}\right| \sqrt{\left|\lambda_{1}\right|^{2}+\left|\lambda_{2}\right|^{2}-\operatorname{Tr}\left(A^{*} J A J\right)}
$$

For the degenerate cases, we may have a singleton, a line, a subset of a line, an open half plane or the whole complex plane.

Proof: i) Let $C=\operatorname{diag}\left(\gamma_{1}, \gamma_{2}\right)$. For any $A \in M_{2}$, we have $V_{J, E_{11}}(A)=V_{J}^{+}(A)$. Since $C=\left(\gamma_{1}+\gamma_{2}\right) E_{11}-\gamma_{2} J$, by properties Q2 and Q4, we get

$$
V_{J, c}(A)=\left(\gamma_{1}+\gamma_{2}\right) V_{J}^{+}(A)-\gamma_{2} \operatorname{Tr}(A J)
$$

If $C$ is a $J$-scalar matrix, it has trace zero and $\operatorname{Tr}(C J)=-2 \gamma_{2}$. Hence

$$
V_{J, c}(A)=\{\operatorname{Tr}(A J) \operatorname{Tr}(C J) / 2\} .
$$

If $C$ is not a $J$-scalar matrix, the result follows from Theorem 3.2. 
ii) For $C=\operatorname{diag}\left(\gamma_{1}, \gamma_{2}\right), A \in M_{2}, U$ a pseudo-unitary matrix in $U_{1,1}$ and

$$
Z=\left[\begin{array}{cc}
0 & -1 \\
1 & 0
\end{array}\right]
$$

the following expansion for the determinant of the sum of two $2 \times 2$ matrices holds:

$$
\operatorname{det}\left(C+U^{*} A U\right)=\operatorname{det}(C)+\operatorname{det}\left(U^{*} A U\right)+\operatorname{Tr}\left(Z^{T} C Z U^{*} A U\right) .
$$

Using this expansion, since $\operatorname{det}(A)=-\operatorname{det}(J A)$ and $Z^{T} C Z=\operatorname{diag}\left(\gamma_{2}, \gamma_{1}\right)$, we have

$$
D_{J, c}(A)=\gamma_{1} \gamma_{2}-\lambda_{1} \lambda_{2}+V_{J, c^{\prime}}(A),
$$

where $c^{\prime}=\left(\gamma_{2}, \gamma_{1}\right)$. That is, the $J, c$-determinantal range of $A$ is obtained from the $J, c^{\prime}$-tracial range by a translation. From $i$ ), it follows that this set is bounded by a branch of a (possibly degenerate) hyperbola, with foci

$$
\begin{aligned}
& \gamma_{1} \gamma_{2}-\lambda_{1} \lambda_{2}+\gamma_{2} \lambda_{1}-\gamma_{1} \lambda_{2}=\left(\gamma_{1}+\lambda_{1}\right)\left(\gamma_{2}-\lambda_{2}\right), \\
& \gamma_{1} \gamma_{2}-\lambda_{1} \lambda_{2}+\gamma_{2} \lambda_{2}-\gamma_{1} \lambda_{1}=\left(\gamma_{1}+\lambda_{2}\right)\left(\gamma_{2}-\lambda_{1}\right)
\end{aligned}
$$

and non-transverse axis of length given in (23).

\section{J,c-Tracial Ranges with Special Shapes}

It is well known that the classical numerical range $W(A)$ of a matrix is a singleton if and only if $A$ is a scalar matrix. Moreover, $W(A) \subseteq \mathbb{R}$ if and only if $A$ is Hermitian. Similar studies have been carried out for the $J$-numerical range [12]. In this section, analogous results are obtained for the $J$, $c$-tracial range, for $J=P\left(I_{r} \oplus-I_{n-r}\right) P^{t}$, with $0 \leq r \leq n$. For simplicity, we will consider in the next sections $P=I_{n}$, since the other cases are obtained in an analogous way.

In the sequel, we adopt the following notation. Let $A \in M_{n}$ and $\alpha \subset$ $\{1, \ldots, n\}$. We denote by $A[\alpha]$ the principal submatrix of $A$ that lies in the rows and columns indexed by $\alpha$.

Lemma 5.1. Let $c=\left(\gamma_{1}, \ldots, \gamma_{n}\right) \in \mathbb{C}^{n}$ and $A=\left(a_{i j}\right) \in M_{n}$. Then $V_{J, c}(A)$ contains the sets

$$
V_{J_{2}, c^{\prime}}\left(A_{11}\right)+\sum_{i \neq k, l} \gamma_{i} a_{i i}
$$

where $c^{\prime}=\left(\gamma_{k}, \gamma_{l}\right), J_{2}=J[k l]$ and $A_{11}=A[k l]$, for $1 \leq k<l \leq n$. 
Proof: Let $C=\operatorname{diag}\left(\gamma_{1}, \ldots, \gamma_{n}\right)$. For $1 \leq k<l \leq n$, consider the principal submatrices of $J, C$ and $A$ that lie in rows and columns $k$ and $l$ : respectively, $J_{2}=J[k l], C_{11}=C[k l]$ and $A_{11}=A[k l]$. Let $M$ be a matrix in $M_{2}$ satisfying $M^{*} J_{2} M=J_{2}$. Now, consider the matrices

$$
A^{\prime}=\left[\begin{array}{ll}
A_{11} & A_{12} \\
A_{21} & A_{22}
\end{array}\right] \quad \text { and } \quad C^{\prime}=\left[\begin{array}{cc}
C_{11} & 0 \\
0 & C_{22}
\end{array}\right]
$$

obtained from $A$ and $C$, respectively, by permuting rows and columns 1 and 2 by rows and columns $k$ and $l$. Let $z$ be an element of the form

$$
z=\operatorname{Tr}\left(C_{11} M^{*} A_{11} M\right)+\sum_{i \neq k, l} \gamma_{i} a_{i i} .
$$

We observe that

$$
\left(M \oplus I_{n-2}\right)^{*} A^{\prime}\left(M \oplus I_{n-2}\right)=\left[\begin{array}{cc}
M^{*} A_{11} M & M^{*} A_{12} \\
A_{21} M & A_{22}
\end{array}\right] .
$$

Consider the matrix $U_{M}$ obtained from $M \oplus I_{n-2}$ by permuting rows and columns 1 and 2 by rows and columns $k$ and $l$, respectively. Easy computations show that

$$
\begin{aligned}
z & =\operatorname{Tr}\left(C_{11} M^{*} A_{11} M\right)+\operatorname{Tr}\left(C_{22} A_{22}\right) \\
& =\operatorname{Tr}\left(C^{\prime}\left(M \oplus I_{n-2}\right)^{*} A^{\prime}\left(M \oplus I_{n-2}\right)\right)=\operatorname{Tr}\left(C U_{M}^{*} A U_{M}\right) .
\end{aligned}
$$

Since $U_{M}$ is a unitary, or a pseudo-unitary, matrix satisfying the condition $U_{M}^{*} J U_{M}=J$, we conclude that $z \in V_{J, c}(A)$.

Theorem 5.1. Let $j_{i}$ denote the ith diagonal element of $J$, and let $c=$ $\left(\gamma_{1}, \ldots, \gamma_{n}\right) \in \mathbb{C}^{n}$, where the $\gamma_{i} j_{i}$ are pairwise distinct, for $i=1, \ldots, n$. For $A \in M_{n}, V_{J, c}(A)$ is a singleton if and only if $A$ is $J$-scalar.

Proof: $(\Leftarrow)$ Let $C=\operatorname{diag}\left(\gamma_{1}, \ldots, \gamma_{n}\right)$. If $A$ is $J$-scalar, then $J A=\xi I_{n}$, for some $\xi \in \mathbb{C}$ and

$$
V_{J, c}(A)=\{\xi \operatorname{Tr}(J C)\}=\{\operatorname{Tr}(J A) \operatorname{Tr}(J C) / n\} .
$$

$(\Rightarrow)$ Now, suppose $A=\left[a_{i j}\right]$ is not $J$-scalar. It is possible to find a principal submatrix $A_{11}=A[k l]$ of $A$, with $1 \leq k<l \leq n$, such that $A_{11}$ is not $J_{2}$-scalar, for $J_{2}=J[k l]$. By Lemma $5.1, V_{J, c}(A)$ contains the subset

$$
\Gamma=V_{J_{2}, c^{\prime}}\left(A_{11}\right)+\sum_{i \neq k, l} \gamma_{i} a_{i i}
$$


where $c^{\prime}=\left(\gamma_{k}, \gamma_{l}\right)$. Since $\gamma_{k} j_{k} \neq \gamma_{l} j_{l}$, the matrix $\operatorname{diag}\left(\gamma_{k}, \gamma_{l}\right)$ is not $J_{2}$-scalar. By the elliptical range theorem concerning $W_{c^{\prime}}\left(A_{11}\right)$, the subset $\Gamma$ of $V_{J, c}(A)$ is an elliptical disc (possibly degenerate) or, by Theorem $4.1 i$ ), a branch of a hyperbola (possibly degenerate) with interior, but never a point. In any case, $V_{J, c}(A)$ is not a singleton.

Theorem 5.2. Let $A \in M_{n}$ and $c=\left(\gamma_{1}, \ldots, \gamma_{n}\right) \in \mathbb{R}^{n}$, with the $\gamma_{i} j_{i}$ pairwise distinct, for $i=1, \ldots, n$. Then $V_{J, c}(A) \subseteq \mathbb{R}$ if and only if $A$ is Hermitian.

Proof: $(\Leftarrow) \operatorname{Let} C=\operatorname{diag}\left(\gamma_{1}, \ldots, \gamma_{n}\right) \in M_{n}(\mathbb{R})$. If $A$ is a Hermitian matrix, then every $z \in V_{J, c}(A)$ satisfies

$$
z=\operatorname{Tr}\left(C U^{*} A U\right)=\operatorname{Tr}\left(C U^{*} A^{*} U\right)=\overline{\operatorname{Tr}\left(C U^{*} A U\right)}=\bar{z},
$$

where $U \in M_{n}$ is such that $U^{*} J U=J$. Therefore, $V_{J, c}(A)$ is a subset of the real line.

$(\Rightarrow)$ Suppose that $A$ is not a Hermitian matrix. Then $A$ contains a nonHermitian principal submatrix, say $A_{11}=A[k l]$, for $1 \leq k<l \leq n$. Using Lemma 5.1 and a similar argument to the one used in the proof of Theorem 5.1 , we find a subset of $V_{J, c}(A)$ that is not contained in the real line, a contradiction.

A matrix $A \in M_{n}$ is said to be essentially $J$-Hermitian if $\mu A+v J$ is Hermitian for some $0 \neq \mu \in \mathbb{C}$ and $v \in \mathbb{C}$.

Theorem 5.3. Let $c=\left(\gamma_{1}, \ldots, \gamma_{n}\right) \in \mathbb{R}^{n}$, with the $\gamma_{i} j_{i}$ pairwise distinct, for $i=1, \ldots, n$, and $A \in M_{n}$. Then $V_{J, c}(A)$ is a subset of a straight line if and only if $A$ is essentially $J$-Hermitian.

Proof: $(\Leftarrow)$ By definition, $A$ is essentially $J$-Hermitian if and only if $\mu A+v J$ is a Hermitian matrix for some $0 \neq \mu \in \mathbb{C}$ and $v \in \mathbb{C}$. By property Q2 and Theorem 5.2, the result follows.

$(\Rightarrow)$ Now, suppose that $V_{J, c}(A)$ is a subset of a straight line. By property Q2, we may rotate and translate $V_{J, c}(A)$, so it becomes a subset of the real line, that is, $V_{J, c}(\mu A+v J) \subseteq \mathbb{R}$, for some $0 \neq \mu \in \mathbb{C}$ and $v \in \mathbb{C}$. By Theorem 5.2, $\mu A+v J$ is Hermitian and so $A$ is essentially $J$-Hermitian.

\section{J,c-Determinantal Ranges with Special Shapes}

Since $\triangle_{C}(A)$ may be seen as the range of a function from $U_{n}$ to $\mathbb{C}, \triangle_{C}(A)$ may be considered a variation on the concept of $W_{C}(A)$. In fact, these two sets have many common properties. As will be seen in the next results as well 
as in their proofs, a certain parallelism still exists between the sets $V_{J, c}(A)$ and $D_{J, c}(A)$.

Lemma 6.1. Let $c=\left(\gamma_{1}, \ldots, \gamma_{n}\right) \in \mathbb{C}^{n}$ and $A=\operatorname{diag}\left(\alpha_{1}, \ldots, \alpha_{n}\right) \in M_{n}$. Then $D_{J, c}(A)$ contains the sets

$$
D_{J_{2}, c^{\prime}}\left(A_{1}\right) \prod_{i \neq k, l}\left(\gamma_{i}+\alpha_{i}\right),
$$

where $c^{\prime}=\left(\gamma_{k}, \gamma_{l}\right), J_{2}=\operatorname{diag}\left(j_{k}, j_{l}\right)$ and $A_{1}=\operatorname{diag}\left(\alpha_{k}, \alpha_{l}\right)$, for $1 \leq k<l \leq n$.

Proof: Let $C=\operatorname{diag}\left(\gamma_{1}, \ldots, \gamma_{n}\right)$. For $1 \leq k<l \leq n$, consider $C_{1}=$ $\operatorname{diag}\left(\gamma_{k}, \gamma_{l}\right)$ and

$$
z=\operatorname{det}\left(C_{1}+M^{*} A_{1} M\right) \prod_{i \neq k, l}\left(\gamma_{i}+\alpha_{i}\right),
$$

with $M$ a matrix in $M_{2}$ satisfying $M^{*} J_{2} M=J_{2}$. The matrix $U_{M}$, obtained from the identity $I_{n}$ by replacing its principal submatrix lying in rows and columns $k$ and $l$ by $M$, satisfies the condition $U_{M}^{*} J U_{M}=J$. Now, let $A_{2}$ and $C_{2}$ be the submatrices obtained from $A$ and $C$, respectively, by deleting rows and columns $k$ and $l$. We easily verify that

$$
z=\operatorname{det}\left(C_{1}+M^{*} A_{1} M\right) \operatorname{det}\left(C_{2}+A_{2}\right)=\operatorname{det}\left(C+U_{M}^{*} A U_{M}\right) \in D_{J, c}(A)
$$

and this completes the proof.

Theorem 6.1. Let $c=\left(\gamma_{1}, \ldots, \gamma_{n}\right) \in \mathbb{C}^{n}$, where the $\gamma_{i} j_{i}$ are pairwise distinct, for $i=1, \ldots, n$, and $A=\operatorname{diag}\left(\alpha_{1}, \ldots, \alpha_{n}\right) \in M_{n}$. Then $D_{J, c}(A)$ is a singleton if and only if $A$ is $J$-scalar.

Proof: $(\Leftarrow)$ Let $C=\operatorname{diag}\left(\gamma_{1}, \ldots, \gamma_{n}\right)$. If $A$ is $J$-scalar, then $A=\xi J$, for some $\xi \in \mathbb{C}$ and

$$
D_{J, c}(A)=\{\operatorname{det}(A+C)\} .
$$

$(\Rightarrow)$ Now, suppose that $A$ is not $J$-scalar. It is possible to find two entries of the matrix $A$, say $\alpha_{k}$ and $\alpha_{l}$, with $1 \leq k<l \leq n$, such that $A_{1}=\operatorname{diag}\left(\alpha_{k}, \alpha_{l}\right)$ is not $J_{2}$-scalar, where $J_{2}=\operatorname{diag}\left(j_{k}, j_{l}\right)$. By Lemma $6.1, D_{J, c}(A)$ contains the subset

$$
\Sigma=D_{J_{2}, c^{\prime}}\left(A_{1}\right) \prod_{i \neq k, l}\left(\gamma_{i}+\alpha_{i}\right)
$$

where $c^{\prime}=\left(\gamma_{k}, \gamma_{l}\right)$. The subset $\Sigma$ of $D_{J, c}(A)$ is either an elliptical disc, by the elliptical range theorem for $\triangle_{c^{\prime}}\left(A_{1}\right)$, or a branch of a hyperbola with 
interior, by Theorem 4.1 ii $)$. As $\operatorname{diag}\left(\gamma_{k}, \gamma_{l}\right)$ is not $J_{2}$-scalar, both sets are possibly degenerate, but they are never a point. In any case, $D_{J, c}(A)$ is not a singleton, contradicting the hypothesis.

Theorem 6.2. Let $c=\left(\gamma_{1}, \ldots, \gamma_{n}\right) \in \mathbb{R}^{n}$, where the $\gamma_{i} j_{i}$ are pairwise distinct, for $i=1, \ldots, n$, and $A=\operatorname{diag}\left(\alpha_{1}, \ldots, \alpha_{n}\right)$. Then $D_{J, c}(A) \subseteq \mathbb{R}$ if and only if $A \in M_{n}(\mathbb{R})$.

Proof: $(\Leftarrow)$ If $A$ is a diagonal matrix in $M_{n}(\mathbb{R})$, then it is clear that $D_{J, c}(A)$ is a subset of the real line.

$(\Rightarrow)$ Suppose that $A$ is a diagonal matrix with at least one non-real principal entry, say $\alpha_{k}$. Then $A$ contains a non-Hermitian principal submatrix, say $A_{1}=\operatorname{diag}\left(\alpha_{k}, \alpha_{l}\right)$. Using Lemma 6.1 and similar arguments to the proof of the previous theorem, we obtain a subset of $D_{J, c}(A)$ that is not contained in the real line, a contradiction. Thus, $A \in M_{n}(\mathbb{R})$.

\section{Special Boundary Points}

Some special boundary points of the numerical ranges have interesting properties. For instance, every non-differentiable boundary point of $W(A)$ is an eigenvalue of $A[3,11]$. Moreover, every corner of $V_{S}(A)$ is an eigenvalue of $S^{-1} A$ (see Theorem 2.1).

In [1] and [2], the corners of $W_{c}(A)$ and $\triangle_{c}(A)$ were investigated. Similar results will be obtained for $V_{J, c}(A)$ and $D_{J, c}(A)$.

Theorem 7.1. Let $A$ and $C$ be matrices in $M_{n}$, such that

$$
C J=\gamma_{1} I_{n_{1}} \oplus \cdots \oplus \gamma_{p} I_{n_{p}}, \quad n_{1}+\cdots+n_{p}=n,
$$

where the $\gamma_{i}$ are pairwise distinct, for $i=1, \ldots, p$, and let $U$ be a pseudounitary matrix in $U_{r, n-r}$. If $z=\operatorname{Tr}\left(C U^{*} A U\right)$ is a corner of $V_{J, C}(A)$, then $U^{*} A U$ is a direct sum $A_{1} \oplus \cdots \oplus A_{p}$, where $A_{i} \in M_{n_{i}}, i=1, \ldots, p$, and

$$
z=\sum_{i=1}^{p} \gamma_{i} \operatorname{Tr}\left(J_{i} A_{i}\right)
$$

where $J_{i} \in M_{n_{i}}, i=1, \ldots, p$, are such that $J=J_{1} \oplus \cdots \oplus J_{p}$.

Proof. For simplicity of notation, write $A_{U}=U^{*} A U$. Since $J=I_{r} \oplus-I_{n-r}$, we have $J^{1 / 2}=I_{r} \oplus i I_{n-r}$. For any Hermitian matrix $S$, and for any $t \in \mathbb{R}$ in a neighborhood of zero, the matrix

$$
e^{i t J^{1 / 2} S J^{1 / 2}}=I+i t J^{1 / 2} S J^{1 / 2}+O\left(t^{2}\right)
$$


is a pseudo-unitary matrix in $U_{r, n-r}$. Consider the differentiable function

$$
f(t)=\operatorname{Tr}\left(C e^{-i t J^{1 / 2 *} S J^{1 / 2 *}} A_{U} e^{i t J^{1 / 2} S J^{1 / 2}}\right),
$$

where $J^{1 / 2 *}=J J^{1 / 2}$. Since $z=\operatorname{Tr}\left(C A_{U}\right)$ is a corner, the derivative of $f$ with respect to $t$ at $t=0$, that is,

$$
\begin{aligned}
f^{\prime}(0) & =i \operatorname{Tr}\left(C A_{U} J^{1 / 2} S J^{1 / 2}-C J^{1 / 2 *} S J^{1 / 2 *} A_{U}\right) \\
& =i \operatorname{Tr}\left(J^{1 / 2} S J^{1 / 2}\left(C A_{U}-J A_{U} C J\right)\right)
\end{aligned}
$$

is zero. Since $S$ is arbitrary, we conclude that

$$
J^{1 / 2}\left(C A_{U}-J A_{U} C J\right) J^{1 / 2}=0
$$

and so $C A_{U}=J A_{U} C J$. Therefore $C J$ commutes with $J A_{U}$, or using the usual notation for the commutator of two matrices

$$
\left[C J, J A_{U}\right]=0,
$$

Since $C J$ is a direct sum of $p$ scalar matrices $\gamma_{i} I_{n_{i}}$, with all the $\gamma_{i}$ distinct, for $i=1, \ldots, p$, from (24) it follows that $A_{U}$ is a direct sum, $A_{1} \oplus \cdots \oplus A_{p}$, where $A_{i} \in M_{n_{i}}, i=1, \ldots, p$. Now, let $J_{i} \in M_{n_{i}}, i=1, \ldots, p$, be such that $J=J_{1} \oplus \cdots \oplus J_{p}$. We easily see that

$$
z=\operatorname{Tr}\left(C J J A_{U}\right)=\sum_{i=1}^{p} \gamma_{i} \operatorname{Tr}\left(J_{i} A_{i}\right)
$$

The following corollary is a straightforward consequence of Theorem 7.1.

Corollary 7.1. Let $C J=I_{k} \oplus 0_{n-k}, 1 \leq k \leq n$, and $A \in M_{n}$. If $z \in$ $V_{J, C}(A)$ is a corner of $V_{J, C}(A)$, then there exists a pseudo-unitary matrix $U \in U_{r, n-r}$ such that $U^{*} A U$ is a direct sum $A_{k} \oplus A_{n-k}$ and $z=\operatorname{Tr}\left(J_{k} A_{k}\right)$, where $J_{k}=J[1 \cdots k]$ and $A_{k} \in M_{k}$.

We observe that Corollary 7.1 is a generalization of Theorem 2.1 on the corners of $V_{J}(A)$. In fact, if $z \in V_{J}(A)$ is a corner of $V_{J}(A)$, by Corollary 7.1 with $k=1$, there exists a pseudo-unitary matrix $U \in U_{r, n-r}$, such that $U^{*} A U=\left[z j_{1}\right] \oplus A_{n-1}$, where $A_{n-1} \in M_{n-1}$. Therefore, $U^{*} A^{*} U=\left[\bar{z} j_{1}\right] \oplus A_{n-1}^{*}$. Then $U^{*} A U e_{1}=z J e_{1}$ and $U^{*} A^{*} U e_{1}=\bar{z} J e_{1}$, where $e_{1}^{*} J e_{1}= \pm 1$. Since $\left(U^{*}\right)^{-1}=J U J$ and $J^{2}=I_{n}$, we have $A U e_{1}=z J U e_{1}$ and $A^{*} U e_{1}=\bar{z} J U e_{1}$, that is, $z$ is an eigenvalue of $J A$ and $x=U e_{1}$ is an eigenvector of $J A$ associated to $z$, such that $x^{*} J x= \pm 1$. 
Theorem 7.2. Let $A$ and $C$ be matrices in $M_{n}$, such that

$$
C J=\gamma_{1} I_{n_{1}} \oplus \cdots \oplus \gamma_{p} I_{n_{p}}, \quad n_{1}+\cdots+n_{p}=n,
$$

where the $\gamma_{i}$ are pairwise distinct, for $i=1, \ldots, p$, and let $U$ be a pseudounitary matrix in $U_{r, n-r}$. If $0 \neq z=\operatorname{det}\left(C+U^{*} A U\right)$ is a corner of $D_{J, C}(A)$, then $U^{*} A U$ is a direct sum of matrices $A_{1} \oplus \cdots \oplus A_{p}$, where $A_{i} \in M_{n_{i}}$, $i=1, \ldots, p$, and

$$
z=\prod_{i=1}^{p} \operatorname{det}\left(\gamma_{i} J_{i}+A_{i}\right)
$$

where $J_{i} \in M_{n_{i}}, i=1, \ldots, p$, are such that $J=J_{1} \oplus \cdots \oplus J_{p}$.

Proof: For simplicity of notation, write $A_{U}=U^{*} A U$. For any Hermitian $S$, consider the differentiable function

$$
f(t)=\operatorname{det}\left(A_{U}+e^{i t J^{1 / 2} S J^{1 / 2}} C e^{-i t J^{1 / 2 *} S J^{1 / 2 *}}\right),
$$

where $J^{1 / 2 *}=J J^{1 / 2}$. The following expansion can be easily obtained

$$
\begin{aligned}
f(t) & =\operatorname{det}\left(A_{U}+C+i t\left(J^{1 / 2} S J^{1 / 2} C-C J^{1 / 2 *} S J^{1 / 2 *}\right)+O\left(t^{2}\right)\right) \\
& =\operatorname{det}\left(A_{U}+C\right)\left[1+i t \operatorname{Tr}\left(\left(A_{U}+C\right)^{-1}\left(J^{1 / 2} S J^{1 / 2} C-C J^{1 / 2 *} S J^{1 / 2 *}\right)\right)\right]+O\left(t^{2}\right),
\end{aligned}
$$

for real $t$ sufficiently close to zero. Since $0 \neq z=\operatorname{det}\left(C+A_{U}\right)$ is a corner, the derivative of $f(t)$ with respect to $t$ at $t=0$ vanishes, that is,

$$
\operatorname{Tr}\left(J^{1 / 2} S J^{1 / 2}\left(C\left(A_{U}+C\right)^{-1}-J\left(A_{U}+C\right)^{-1} C J\right)\right)=0 .
$$

Since $S$ is arbitrary, we obtain

$$
J C\left(A_{U}+C\right)^{-1}=\left(A_{U}+C\right)^{-1} C J .
$$

This last equation implies

$$
\left(A_{U}+C\right) J C J=C J\left(A_{U}+C\right) J
$$

and so $C J$ commutes with $A_{U} J$ and the result follows.

The analysis of the $J, c$-tracial range for $2 \times 2$ matrices leads to the characterization of those matrices $A \in M_{n}$ for which $V_{J, c}(A)$ is a singleton or a subset of a straight line. The analysis of the $2 \times 2$ case is also useful in the study of some special boundary points of the $J, c$-tracial range.

Theorem 7.3. Let $c=\left(\gamma_{1}, \ldots, \gamma_{n}\right) \in \mathbb{C}^{n}$ and $A=\left(a_{k l}\right)$ be an upper triangular matrix in $M_{n}$, with diagonal elements $\alpha_{1}, \ldots, \alpha_{n}$. If $\sum_{i=1}^{n} \gamma_{i} \alpha_{i}$ is a boundary point of $V_{J, c}(A)$ and $\gamma_{k} j_{k} \neq \gamma_{l} j_{l}$, then $a_{k l}=0$, for $1 \leq k<l \leq n$. 
Proof: Let $1 \leq k<l \leq n$, such that $\gamma_{k} j_{k} \neq \gamma_{l} j_{l}$. Consider the following $2 \times 2$ principal submatrix of $A$,

$$
A_{11}=\left[\begin{array}{cc}
\alpha_{k} & a_{k l} \\
0 & \alpha_{l}
\end{array}\right],
$$

where $a_{k l} \neq 0$. By Lemma 5.1, $V_{J, c}(A)$ contains the subset

$$
\Gamma=V_{J_{2}, c^{\prime}}\left(A_{11}\right)+\sum_{i \neq k, l} \gamma_{i} \alpha_{i},
$$

where $c^{\prime}=\left(\gamma_{k}, \gamma_{l}\right)$ and $J_{2}=J[k l]$. If $j_{k}=j_{l}$, then $V_{J_{2}, c^{\prime}}\left(A_{11}\right)$ is an elliptical disc, by the elliptical range theorem for $W_{c^{\prime}}\left(A_{11}\right)$. If $j_{k}=-j_{l}$, then by Theorem 4.1 i) $V_{J_{2}, c^{\prime}}\left(A_{11}\right)$ is a branch of a hyperbola with interior. Since $A_{11}$ is not Hermitian and $\operatorname{diag}\left(\gamma_{k}, \gamma_{l}\right)$ is not $J_{2}$-scalar, these conics have a focus at $\gamma_{k} \alpha_{k}+\gamma_{l} \alpha_{l}$ and may degenerate either into a circular disc, a half plane or the whole complex plane; in any event, the focus is an interior point. In any case, the subset $\Gamma$ of $V_{J, c}(A)$ has an interior point at $\sum_{i=1}^{n} \gamma_{i} \alpha_{i}$, which contradicts the assumption of this point being on the boundary of $V_{J, c}(A)$, unless $a_{k l}$ vanishes.

The following two corollaries are obvious consequences of this theorem.

Corollary 7.2. Let $A$ be an upper triangular matrix in $M_{n}$, and let

$$
C J=\gamma_{1} I_{n_{1}} \oplus \cdots \oplus \gamma_{p} I_{n_{p}}, \quad n_{1}+\cdots+n_{p}=n,
$$

where the $\gamma_{i} j_{i}$ are pairwise distinct, for $i=1, \ldots, p$. If $\operatorname{Tr}(C A)$ is a boundary point of $V_{J, C}(A)$, then $A=A_{1} \oplus \cdots \oplus A_{p}$, where each block $A_{i}$ is an upper triangular matrix in $M_{n_{i}}, i=1, \ldots, p$.

Corollary 7.3. Let $c=\left(\gamma_{1}, \ldots, \gamma_{n}\right) \in \mathbb{C}^{n}$, with the $\gamma_{i} j_{i}$ pairwise distinct, for $i=1, \ldots, p$, and let $A$ be an upper triangular matrix in $M_{n}$ with diagonal elements $\alpha_{1}, \ldots, \alpha_{n}$. If $\sum_{i=1}^{n} \gamma_{i} \alpha_{i}$ is a boundary point of $V_{J, c}(A)$, then $A$ is a diagonal matrix.

\section{Algorithm and Examples}

The classical numerical range and its generalizations have been extensively studied and many algorithms and computer programs for generating these sets have been presented $[10,15]$. In this section, we describe an algorithm that will be used to plot an approximation for the $J$-numerical range. 
In [13], a Matlab program for generating the $S$-numerical range $W_{S}^{+}(A)$, for $S$ a Hermitian matrix, has been developed. After generating the joint numerical range of the Hermitian matrix triple $(H, G, S)$, with $S A=H+$ $i G$, the points $(x / z, y / z)$, where $(x, y, z) \in W(H, G, S)$ and $z>0$, were plotted. From such a collection of points, an approximation for $W_{S}^{+}(A)$ was easily obtained. Now, we generate the $J$-numerical range, using a different approach.

Basically, the idea of the algorithm is to compute the eigenvalues of $B_{s}=$ $J \operatorname{Re}\left(e^{i \theta_{s}} A\right)$, where $\theta_{s}$ varies on a specified subset of $[0,2 \pi]$. If there exist directions $e^{i \theta_{s}}$, for which not all the eigenvalues of $B_{s}$ are real, then no support lines exist in those directions. Therefore, we search for those directions $e^{i \theta_{s}}$ for which all the eigenvalues of $B_{s}$ are real. We also make sure that the $J$-norm of the corresponding eigenvectors is different from 0 . In these circumstances, the boundary generating curves of $V_{J}(A)$ exist. By the $p$-convexity of the $J$ numerical range, the representation of $V_{J}(A)$ is readily obtained. Otherwise, this set is a line (in certain cases a subset of a line), or the whole complex plane (in some cases, without a line).

Firstly, we present an algorithm providing the boundary generating curve of the $J$-numerical range. Matlab programs have been written to plot this curve and to draw an approximation for $V_{J}(A)$.

\section{Figure 1}

Step 1: Consider the directions $e^{i \theta_{s}}$, where

$$
\theta_{s}=\frac{\pi(s-1)}{2 m}, \quad s=1, \ldots, 4 m+1,
$$

for some positive integer $m \geq 20$. For $A \in M_{n}$ and for each choice of $s$, compute the eigenvalues of $B_{s}=J \operatorname{Re}\left(e^{i \theta_{s}} A\right)$. If these eigenvalues are all real and the $J$-norm of the corresponding eigenvectors is not 0 , put the value $s$ in a vector denoted by direc $=\left(t_{1}, \ldots, t_{k}\right)$. Observe that the number of eigenvectors with positive $J$-norm is fixed by the inertia of $J$, independently of $\theta_{s}$.

Step 2: If the vector direc is empty, the following cases are possible:

(i) If $A$ is Hermitian, then $V_{J}(A)=\mathbb{R}$; 

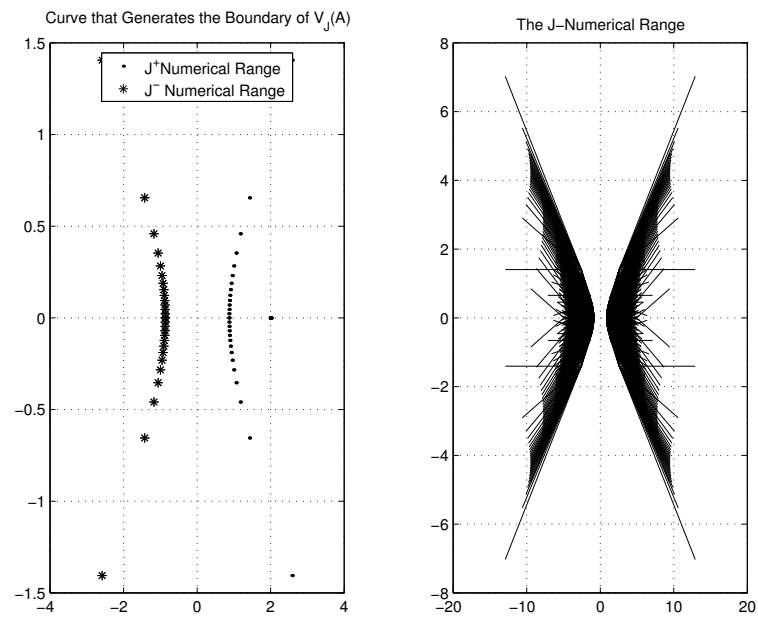

(ii) If $A$ is essentially $J$-Hermitian, that is, $A=\alpha S+\beta J$, for some Hermitian matrix $S \in M_{n}, 0 \neq \alpha \in \mathbb{C}$ and $\beta \in \mathbb{C}$, then $V_{J}(A)$ is a line passing through $\beta$ and with direction $\arg \alpha$;

(iii) Otherwise, $V_{J}(A)$ is the whole complex plane, possibly without a line.

Figure 2
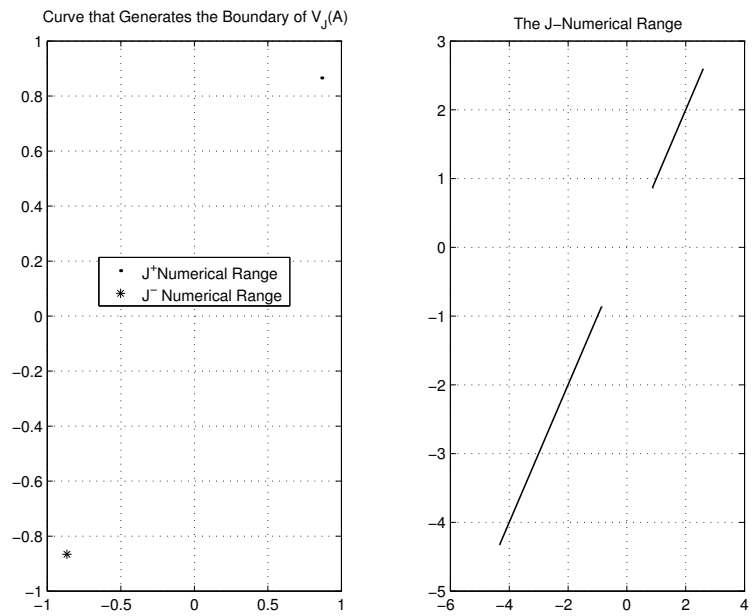
Step 3: If the situations referred to in the previous step do not occur, then for each entry $t_{j}$ of the vector direc, compute $n$ linearly independent eigenvectors associated with the eigenvalues of $B_{t_{j}}$. (The linear independence of the eigenvectors ensures that all the generating boundary curves are considered.) Denote these eigenvectors by $u_{i}\left(t_{j}\right)$, for $i=1, \ldots, n$, and evaluate

$$
\frac{u_{i}^{*}\left(t_{j}\right) A u_{i}\left(t_{j}\right)}{u_{i}^{*}\left(t_{j}\right) J u_{i}\left(t_{j}\right)}
$$

for $u_{i}^{*}\left(t_{j}\right) J u_{i}\left(t_{j}\right)>0$ and $u_{i}^{*}\left(t_{j}\right) J u_{i}\left(t_{j}\right)<0$. Suppose that $r$ of these eigenvectors have positive $J$-norm while $n-r$ have negative $J$-norm. Then $r$ points in $V_{J}^{+}(A)$ and $n-r$ points in $-V_{J}^{-}(A)$ are generated. Thus, for all the $t_{j}$, $j=1, \ldots, k, r k$ points in $V_{J}^{+}(A)$ (possibly coincident) and $(n-r) k$ points in $-V_{J}^{-}(A)$ (possibly coincident) are represented.

To generate an approximation of $V_{J}(A)$ we proceed as follows.

Step 4: For any distinct points $x, y \in V_{J}^{+}(A)$, compute $\alpha x+(1-\alpha) y$, for $0 \leq \alpha \leq 1$. Repeat this step for $-V_{J}^{-}(A)$. The algorithm draws $\left(\begin{array}{c}r k \\ 2\end{array}\right)$ line segments connecting points in $V_{J}^{+}(A)$ and $\left(\begin{array}{c}n k-r k \\ 2\end{array}\right)$ line segments between points in $-V_{J}^{-}(A)$.

Step 5: For any distinct points $x \in V_{J}^{+}(A)$ and $y \in-V_{J}^{-}(A)$, compute $\alpha x+(1-\alpha) y$, for $\alpha \leq 0$ or $\alpha \geq 1$. Here, the number of lines is $r(n-r) k^{2}$.

The set obtained in Step 4 and Step 5 is the pseudo-convex hull of the boundary generating curves of $V_{J}(A)$.

In some cases, the Matlab program, instead of plotting $V_{J}(A)$, may present a message describing the shape of the set. This is the case when $A$ is essentially $J$-Hermitian and the boundary generating curve of $V_{J}(A)$ does not exist; nevertheless, for all the other cases of essentially $J$-Hermitian matrices a plot is always given (see Figure 2).

When $V_{J}(A)$ is the whole complex plane an approximation for $V_{J}(A)$ is plotted, being clearly seen that this set is $\mathbb{C}$ (see Figure 3 ).

Now, some examples are considered. 
Figure 3
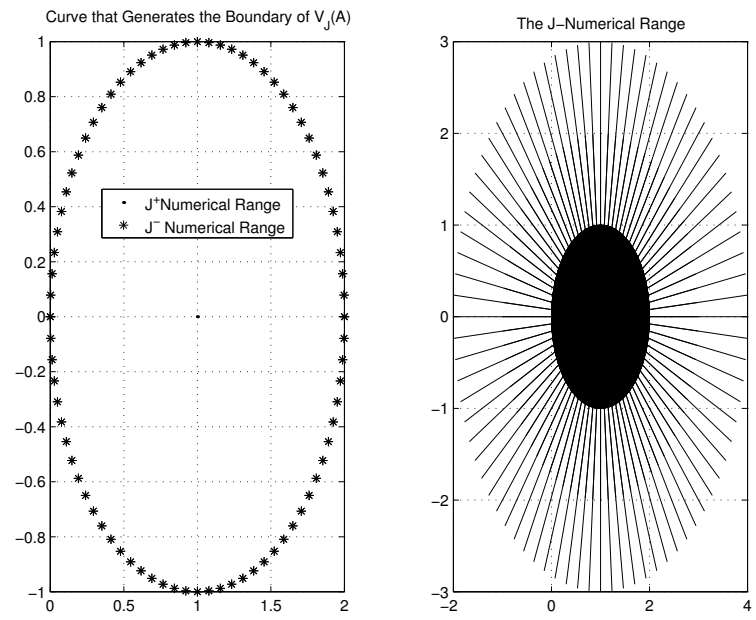

Example 1: Let $J=\operatorname{diag}(1,-1,1)$ and

$$
A=\left[\begin{array}{lll}
1 & 0 & a \\
0 & b & 0 \\
0 & 0 & 1
\end{array}\right],
$$

with $a, b \in \mathbb{R}$. We easily check that if $1-\frac{1}{2}|a| \leq-b \leq 1+\frac{1}{2}|a|, V_{J}(A)$ is the whole complex plane. Otherwise, the boundary generating curves of $V_{J}(A)$ are the singleton $\{-b\}$ and the circle

$$
(x-1)^{2}+y^{2}=\frac{a^{2}}{4}
$$

(more precisely, two superimposed circles). Hence, $V_{J}(A)$ is the pseudoconvex hull of these curves.

Example 2: Consider $J=\operatorname{diag}(-1,1,1)$ and the same matrix $A$ of the previous example. It can be easily seen that if either $\frac{a^{2}}{4} \geq 1$ or $-1+\frac{a^{2}}{4} \geq b$, then $V_{J}(A)$ reduces to the whole complex plane. Otherwise, the singleton $\{b\}$ and the two branches of the hyperbola

$$
\frac{x^{2}}{1-\frac{a^{2}}{4}}-\frac{y^{2}}{\frac{a^{2}}{4}}=1
$$

are the boundary generating curves of $V_{J}(A)$, which is the pseudo-convex hull of these curves. 
Figure 4
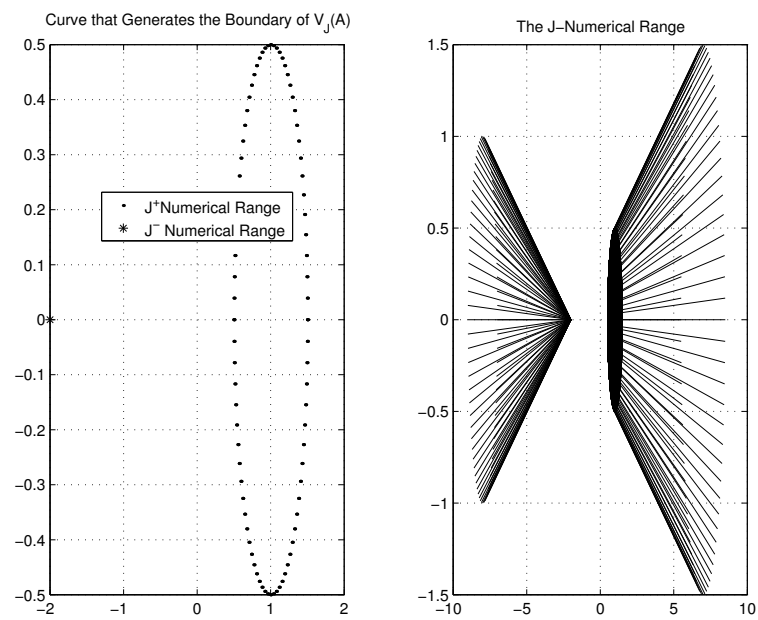

Figure 1 and Figure 4 represent $V_{J}(A)$ for the matrices in the Examples 2 and 1 , respectively, in the particular case $a=1$ and $b=2$.

Example 3: Consider, now, $J=\operatorname{diag}(1,-1,-1)$ and

$$
A=\left[\begin{array}{ccc}
1 & 0 & 0 \\
0 & -1 & b \\
0 & 0 & -1
\end{array}\right],
$$

with $b \in \mathbb{R}$. The boundary generating curves of $V_{J}(A)$ are the singleton $\{1\}$ and the circle

$$
(x-1)^{2}+y^{2}=\frac{b^{2}}{4} .
$$

For the particular case $b=2$, see Figure 3. In this example, the pseudoconvex hull of these curves gives the whole complex plane.

The eigenvalues of $J \operatorname{Re}\left(e^{i \theta} A\right), \theta \in \mathbb{R}$, when $b=2$, are the real numbers $\cos \theta$ and $\cos \theta \pm 1$. Nevertheless, $V_{J}(A)$ does not have support lines in any direction, and so the converse of Proposition 2.1 does not hold.

Example 4: Let $J=\operatorname{diag}(1,-1)$ and $A$ be the following essentially $J$ Hermitian matrix:

$$
A=(1+i)\left[\begin{array}{ll}
1 & a \\
a & 1
\end{array}\right]
$$


with $a>0$. If $a>1$, then $V_{J}(A)=e^{i \pi / 4} \mathbb{R}$ and if $a=1$, then $V_{J}(A)=$ $e^{i \pi / 4} \mathbb{R} \backslash\{0\}$. If $0<a<1$, the boundary generating curve of $V_{J}(A)$ is formed by the points $\pm e^{i \pi / 4} \sqrt{1-a^{2}}$. For the particular case $a=1 / 2, V_{J}(A)$ is the line defined by $\pm e^{i \pi / 4} \sqrt{3} / 2$, except the open line segment joining these points (see Figure 2).

\section{Acknowledgment}

The authors are very grateful to the Referee for his careful reading of the manuscript and for valuable suggestions.

\section{References}

[1] N. Bebiano, Nondifferentiable points of $\partial W_{c}(A)$, Linear and Multilinear Algebra 19 (1986), 249-257.

[2] N. Bebiano, Some analogies between the $c$-numerical range and a certain variation on this concept, Linear Algebra Appl. 81 (1986), 47-54.

[3] W. F. Donoghue, On the numerical range of a bounded operator, Michigan Math. J. 4 (1957), 261-263.

[4] A. L. Duarte, O Teorema de Tarski e suas Aplicações em Teoria de Matrizes, Universidade de Coimbra, 1993.

[5] M. Goldberg and E. G. Straus, Elementary inclusion relations of generalized numerical ranges, Linear Algebra Appl. 18 (1977), 1-24.

[6] R. D. Grigorieff and R. Platto, On the minimax equality for seminorms, Linear Algebra Appl. 221 (1995), 227-243.

[7] R. A. Horn and C. R. Johnson, Matrix Analysis, Cambridge University Press, New York, 1985.

[8] R. A. Horn and C. R. Johnson, Topics in Matrix Analysis, Cambridge University Press, New York, 1991.

[9] N. Jacobson, Basic Algebra, Freeman, 1979.

[10] C. R. Johnson, Numerical determinantion of the field of values of a general complex matrix, SIAM J. Numer. Anal 15 (1978), 595-602.

[11] R. Kippenhahn, Uber der wertevorrat einer matrix, Math. Nach. 6 (1951), 193-228.

[12] C.-K. Li, N. K. Tsing and F. Uhlig, Numerical ranges of an operator in an indefinite inner product space, Electr. J. Linear Algebra 1 (1996), 1-17.

[13] C.-K. Li and L. Rodman, Shapes and computer generation of numerical ranges of Krein space operators, Electr. J. Linear Algebra 3 (1998), 31-47.

[14] C.-K. Li and L. Rodman, Remarks of numerical ranges of operators in spaces with an indefinite inner product, Proc. Amer. Math. Soc. 126 (1998), 973-982.

[15] M. Marcus and C. Pesce, Computer generated numerical ranges and some resulting theorems, Linear and Multilinear Algebra Appl. 20 (1987), 121-157.

[16] F. D. Murnaghan, On the field of values of a square matrix Proc. Nat. Acad. Sci. 18 (1932), 246-248 
[17] H. Nakazato, Y. Nishikawa and M. Takaguchi, On the boundary of the $C$-numerical range of a matrix, Linear Algebra Appl. 39 (1995), 231-240.

[18] H. Shapiro, A conjecture of Kippenhahn about the characteristic polynomial of a pencil generated by two Hermitian matrices. I, Linear Algebra Appl. 43 (1982), 201-221.

[19] H. Shapiro, A conjecture of Kippenhahn about the characteristic polynomial of a pencil generated by two Hermitian matrices. II, Linear Algebra Appl. 45 (1982), 97-108.

N. BEBIANO

University of Coimbra, Mathematics Department, P 3000 Coimbra

R. LEMOS

University of Aveiro, Mathematics Department, P 3810-193 Aveiro

J. DA PROVIDÊNCIA

University of Coimbra, Physics Department, P 3000 Coimbra

G. SOARES

University of Trás-os-Montes and Alto Douro, Mathematics Department, P 5000 Vila REAL 